\title{
INVERSE SCATTERING OF ELASTIC WAVES BY PERIODIC STRUCTURES: UNIQUENESS UNDER THE THIRD OR FOURTH KIND BOUNDARY CONDITIONS*
}

\author{
JOHANNES ELSCHNER ${ }^{\dagger}$ AND GUANGHUI HU ${ }^{\dagger}$
}

\begin{abstract}
The inverse scattering of a time-harmonic elastic wave by a two-dimensional periodic structure in $\mathbb{R}^{2}$ is investigated. The grating profile is assumed to be the graph of a continuous piecewise linear function on which the third or fourth kind boundary conditions are satisfied. Via an equivalent variational formulation, existence of quasi-periodic solutions for general Lipschitz grating profiles is proved by applying the Fredholm alternative. However, uniqueness of solution to the direct problem does not hold in general. For the inverse problem, we determine and classify all the unidentifiable grating profiles corresponding to a given incident elastic field, relying on the reflection principle for the Navier equation and the rotational invariance of propagating directions of the total field. Moreover, global uniqueness for the inverse problem is established with a minimal number of incident pressure or shear waves, including the resonance case where Rayleigh frequencies are allowed. The gratings that are unidentifiable by one incident elastic wave provide non-uniqueness examples for appropriately chosen wave number and incident angles.
\end{abstract}

Key words. Diffraction gratings, inverse scattering, uniqueness, elastic waves.

AMS subject classifications. 78A46, 35B27, 35R30, 74B05.

1. Introduction. Assume a time-harmonic (with time variation of the form $\exp (-i \omega t), \omega>0)$ incident plane elastic wave is scattered by a diffraction grating in a linear isotropic and homogeneous elastic medium. Suppose further that the grating is periodic in $x_{1}$-direction and invariant in $x_{3}$-direction, and that all elastic waves are propagating perpendicular to the $x_{3}$-axis, so that the problem can be treated as a problem of plane elasticity. Moreover, the diffraction grating is supposed to have an impenetrable surface on which normal displacement and tangential stress (or normal stress and tangential displacement) vanish. This gives rise to the so-called third (or fourth) kind boundary conditions for the Navier equation. The direct problem is to predict the displacement distribution given the incident elastic waves and grating profile, whereas the inverse problem is to determine the grating profile from nearfield measurements on a straight line above the grating. To the authors' knowledge, there does not exist any result regarding the direct and inverse scattering of elastic waves by diffraction gratings under the third or fourth kind boundary conditions. The aim of this paper is to fill these gaps. We refer to the monograph [20] for a comprehensive treatment of the boundary value problem of elasticity (including the boundary conditions of the third and fourth kinds) and the monograph [8] for applications of diffraction gratings.

There exist several solvability results for the direct scattering problem when the displacement vanishes on the grating surfaces ( which is called the first kind or Dirichlet boundary condition). Based on the boundary integral equation method, T. Arens (see [3]) established the existence and uniqueness of quasi-periodic solutions for the Dirichlet problem if the grating profile is given by the graph of a smooth $\left(C^{2}\right)$ function; see also [4] for the investigation of the Green's tensor of the Navier equation for a half-space and [5], [6] for more general rough surface scattering problems. The same

\footnotetext{
*Received July 14, 2010; accepted for publication February 26, 2011.

${ }^{\dagger}$ Weierstrass Institute for Applied Analysis and Stochastics, Mohrenstr. 39, 10117 Berlin, Germany (\{elschner; hu\}@wias-berlin.de).
} 
Dirichlet problem in general Lipschitz domains is investigated by Elschner \& Hu [13] but via a variational method. It is shown in [13] that, for either an incident plane pressure or shear wave, there always exists a quasi-periodic solution to the direct problem by establishing the strong ellipticity of the corresponding variational formulation over a bounded periodic cell and then applying the Fredholm alternative. Moreover, uniqueness can be guaranteed if the grating profile is given by a Lipschitz graph. In Section 3 of this paper, we will review the existence proof of [13] and show that it is also valid for more general incident elastic waves when the boundary conditions of the third or fourth kind are imposed on the grating profiles. However, uniqueness does not hold in general since the solution scattered by a flat grating is not unique if a Rayleigh frequency occurs; see Theorem 3.

We then proceed to consider uniqueness for the inverse problem which involves near-field measurements. To our knowledge, the only paper dealing with the inverse scattering of elastic waves by diffraction gratings is [2], which extends Hettlich and Kirsch's work on Schiffer's theorem (see [18]) to the case of inverse elastic diffraction problems. It is proved in [2] that a Dirichlet periodic smooth surface $\left(C^{2}\right)$ can be uniquely determined from the scattered field for one incident pressure wave and an interval of wave numbers. Furthermore, a finite set of wave numbers is enough if a priori information about the height of the grating curve is known. For other uniqueness results within smooth periodic profiles, we refer to [7], [19], [1] and [11] for the inverse scattering of acoustic or electromagnetic waves. It should be mentioned that, if the grating profiles are piecewise linear, by the reflection principles for the Helmholtz and Maxwell equations, global uniqueness results by several incident waves are available for the inverse scattering of time-harmonic electromagnetic waves, including TE or TM polarization; see [9], [10], [14], [15] and [16]. Relying on the reflection principle for the Navier system developed in [17], in this paper we are aimed to prove global uniqueness for the inverse elastic diffraction problem in the case of boundary conditions of the third or fourth kind. Note that such an approach does not work under the first kind (Dirichlet) or second kind (Neumann) boundary condition, since in these cases there seems to be no reflection principle.

It is demonstrated in Section 4 that the global uniqueness by a fixed number of incident pressure or shear waves is impossible for determining a flat grating. Thus, such gratings should not be included in the admissible class $\mathcal{A}$ of grating profiles, which, in this paper, are given by the graphs of piecewise continuous linear functions. Following the arguments in [15], we prove that the total fields generated by two different grating profiles of $\mathcal{A}$ but taking the same near-field values can be reduced to a finite sum of propagating waves; see Lemma 3 in Section 5 . Then, inspired by the ideas in [9],[10] and [14], we obtain the rotational invariance of the reduced total field, using the reflection principle for the Navier equation; see Lemma 7. By using the rotational invariance, we can determine all classes of grating profiles of $\mathcal{A}$ that cannot be uniquely identified from the knowledge of the scattered waves corresponding to only one incident wave. This enables us to prove global uniqueness within the polygonal periodic structures by a minimal number of incident waves. Our main results on the inverse problem are

- Under the boundary conditions of the fourth kind, two incident pressure waves (four incident shear waves) are enough to uniquely determine a grating $\Lambda \in \mathcal{A}$, while one incident pressure wave is (three incident shear waves are) sufficient if Rayleigh frequencies for the compressional (shear) part of the displacement are excluded. 
- Under the boundary conditions of the third kind, four incident pressure waves (two incident shear waves) are enough to uniquely determine a grating $\Lambda \in \mathcal{A}$, while three incident pressure waves are (one incident shear wave is) sufficient if Rayleigh frequencies for the compressional (shear) part of the displacement are excluded.

Note that the above two results are similar to those in [16] on inverse scattering of electromagnetic waves for TE and TM polarizations.

The paper is organized as follows. In Section 2, we rigorously formulate the direct and inverse problems. Following [3] and [13], a radiation condition based on Rayleigh expansions is used in the direct problem. In Section 3, we investigate the existence of quasi-periodic solutions for a broad class of incident elastic waves in general Lipschitz domains and present non-uniqueness examples for the direct diffraction problem. The reflection principle for the Navier equation together with the reduction of the total field to a finite number of terms is presented in Section 5. The aim of Section 6 is to prove global uniqueness with a minimal number of incident pressure waves in the resonance case. Compared to the case where Rayleigh frequencies are excluded (see [9] for the inverse scattering of electromagnetic waves), the resonance case gives rise to additional classes of unidentifiable grating profiles which provide non-uniqueness examples for appropriately chosen wave number and incident angles. Finally, in Section 7, we apply the arguments from Section 6 to prove uniqueness for the incident shear waves.

Throughout the paper, we assume that a grating profile is always given by the graph of some continuous piecewise linear function, which excludes the case of piecewise constant profile functions. However, the method presented in Section 6 can be extended to prove uniqueness for an arbitrary polygonal grating profile. To this end, we need to justify the first assertion of Lemma 3 using the path argument developed recently for bounded obstacle scattering problems (see, e.g., [17, 21, 23]), and then determine the compressional and shear parts of the total field using rotational invariance. Note that Lemma 8 would not remain true if the grating profile contained line segments parallel to the $x_{2}$-axis. The idea of proving rotational invariance can be employed to simplify the uniqueness proof in [14].

We believe that all the arguments within this paper can be extended to the 3D case of doubly periodic structures using a single incident pressure or shear wave. Note that the diffraction problem in $\mathbb{R}^{3}$ can be reduced to a problem of plane elasticity under the additional assumptions that the three-dimensional grating varies only in $x_{1}$ and remain invariant in $x_{3}$ and that all elastic waves are propagating perpendicular to the $x_{3}$-axis. Thus, it is quite natural to view the unidentifiable grating curves in the $\left(x_{1}, x_{2}\right)$-plane as non-uniqueness examples for the inverse scattering by bi-periodic structures in $\mathbb{R}^{3}$. Nevertheless, we still need to consider the three-dimensional gratings which vary in both $x_{1}$ and $x_{2}$ and the case where the incident wave is not perpendicular to the $x_{3}$-axis.

2. Mathematical formulation of the direct and inverse scattering problems. Let the cross-section of the diffraction grating in the $\left(x_{1}, x_{2}\right)$-plane be given by a Lipschitz curve $\Lambda$, which is $2 \pi$-periodic with respect to $x_{1}$-direction. The region above the grating is denoted by $\Omega_{\Lambda}$. Suppose that a time-harmonic plane elastic wave with the incident angle $\theta \in(-\pi / 2, \pi / 2)$ is incident on $\Lambda$ from above, which is either an incident pressure wave taking the form

$$
u^{i n}=u_{p}^{i n}(x)=\hat{\theta} \exp \left(i k_{p} x \cdot \hat{\theta}\right) \quad \text { with } \quad \hat{\theta}:=(\sin \theta,-\cos \theta)^{\top}
$$


or an incident shear wave taking the form

$$
u^{i n}=u_{s}^{i n}(x)=\hat{\theta}^{\perp} \exp \left(i k_{s} x \cdot \hat{\theta}\right) \quad \text { with } \quad \hat{\theta}^{\perp}:=(\cos \theta, \sin \theta)^{\top},
$$

where

$$
k_{p}:=\omega / \sqrt{2 \mu+\lambda}, \quad k_{s}:=\omega / \sqrt{\mu}
$$

are the compressional and shear wave numbers respectively, $\lambda$ and $\mu$ are the Lamé constants satisfying $\mu>0$ and $\lambda+\mu>0, \omega>0$ denotes the angular frequency of the harmonic motion and $T$ indicates the transpose of a vector in $\mathbb{R}^{2}$. For simplicity we assume the mass density of the elastic medium is equal to one, so that the total displacement $u\left(x_{1}, x_{2}\right)$, which can be decomposed as the sum of the incident field $u^{i n}$ and the scattered field $u^{s c}$, satisfies the Navier equation (or system):

$$
\left(\Delta^{*}+\omega^{2}\right) u=0 \quad \text { in } \quad \Omega_{\Lambda}, \quad \Delta^{*}:=\mu \Delta+(\lambda+\mu) \operatorname{grad} \operatorname{div} .
$$

We assume the grating is impenetrable, and the vanishing normal displacement and tangential stress (or normal stress and tangential displacement) lead to the following boundary conditions on $\Lambda$ :

$$
\text { boundary conditions of the third kind: } \mathbf{n} \cdot u=0, \tau \cdot T u=0 \text {, }
$$$$
\text { or boundary conditions of the fourth kind: } \tau \cdot u=0, \mathbf{n} \cdot T u=0 \text {, }
$$

where $T u$ stands for the stress vector or traction having the form:

$$
T u=2 \mu \partial_{\mathbf{n}} u+\lambda \mathbf{n} \operatorname{div} u+\mu \tau\left(\partial_{2} u_{1}-\partial_{1} u_{2}\right)
$$

with the exterior unit normal $\mathbf{n}=\left(n_{1}, n_{2}\right)^{\top}$ and the unit tangential vector $\tau=$ $\left(-n_{2}, n_{1}\right)^{\top}$ on $\Lambda$. Here and in the following the notation $\partial_{j} v=\frac{\partial v}{\partial x_{j}}$ is used. The periodicity of the structure together with the form of the incident waves implies that the solution $u$ must be quasi-periodic with the phase-shift $\alpha$ (or $\alpha$-quasi-periodic), i.e.

$$
u\left(x_{1}+2 \pi, x_{2}\right)=\exp (2 i \alpha \pi) u\left(x_{1}, x_{2}\right), \quad\left(x_{1}, x_{2}\right) \in \Omega_{\Lambda},
$$

where either $\alpha:=k_{p} \sin \theta$ for the incident pressure wave (1), or $\alpha:=k_{s} \sin \theta$ for the incident shear wave (2). To ensure well-posedness of the boundary value problem (3)-(7), a radiation condition must be imposed as $x_{2} \rightarrow+\infty$. Note that the scattered field $u^{s c}$, which also satisfies the Navier equation (3), can be decomposed into its compressional and shear parts,

(8) $u^{s c}=\frac{1}{i}(\operatorname{grad} \varphi+\overrightarrow{\operatorname{curl}} \psi) \quad$ with $\varphi:=-\frac{i}{k_{p}^{2}} \operatorname{div} u^{s c}, \quad \psi:=\frac{i}{k_{s}^{2}} \operatorname{curl} u^{s c}$,

where the two curl operators in $\mathbb{R}^{2}$ are defined by

$$
\text { curl } u:=\partial_{1} u_{2}-\partial_{2} u_{1}, \quad u=\left(u_{1}, u_{2}\right)^{\top} \text { and } \quad \overrightarrow{\operatorname{curl}} v:=\left(\partial_{2} v,-\partial_{1} v\right)^{\top},
$$

and the scalar functions $\varphi, \psi$ satisfy the homogeneous Helmholtz equations

$$
\left(\Delta+k_{p}^{2}\right) \varphi=0 \text { and }\left(\Delta+k_{s}^{2}\right) \psi=0 \quad \text { in } \quad \Omega_{\Lambda} .
$$


Applying the Rayleigh expansion for the scalar Helmholtz equation to $\varphi$ and $\phi$ respectively, we finally obtain a corresponding expansion of $u^{s c}$ into outgoing plane elastic waves:

(10) $u^{s c}(x)=$

$$
\sum_{n \in \mathbb{Z}}\left\{A_{p, n}\left(\begin{array}{c}
\alpha_{n} \\
\beta_{n}
\end{array}\right) \exp \left(i \alpha_{n} x_{1}+i \beta_{n} x_{2}\right)+A_{s, n}\left(\begin{array}{c}
\gamma_{n} \\
-\alpha_{n}
\end{array}\right) \exp \left(i \alpha_{n} x_{1}+i \gamma_{n} x_{2}\right)\right\}
$$

for $x_{2}>\Lambda^{+}$, where the constants $A_{p, n}, A_{s, n} \in \mathbb{C}$ are called the Rayleigh coefficients and

(11) $\Lambda^{+}:=\max _{\left(x_{1}, x_{2}\right) \in \Lambda} x_{2}, \quad \alpha_{n}:=\alpha+n, \quad \beta_{n}=\beta_{n}(\theta):=\left\{\begin{array}{l}\sqrt{k_{p}^{2}-\alpha_{n}^{2}} \\ i \sqrt{\alpha_{n}^{2}-k_{p}^{2}} \quad \text { if } \quad\left|\alpha_{n}\right| \leq k_{p} \mid>k_{p},\end{array}\right.$

and $\gamma_{n}:=\gamma_{n}(\theta)$ is defined analogously as $\beta_{n}$ with $k_{p}$ replaced by $k_{s}$. This is the radiation condition we are going to use in the following; see also [3]. Since $\beta_{n}$ and $\gamma_{n}$ are real for at most a finite number of indices, only a finite number of plane waves in (10) propagate into the far field, with the remaining evanescent waves (or surface waves) decaying exponentially as $x_{2} \rightarrow+\infty$. The above expansion converges uniformly with all derivatives in the half-plane $\left\{x \in \mathbb{R}^{2}: x_{2} \geq b\right\}$ for any $b>\Lambda^{+}$. Given a fixed incident angle $\theta$, define

$$
\pi_{p}:=\left\{n \in \mathbb{Z}: \beta_{n}(\theta)=0\right\}, \quad \pi_{s}:=\left\{n \in \mathbb{Z}: \gamma_{n}(\theta)=0\right\} .
$$

We say that a Rayleigh frequency occurs if either $\pi_{p} \neq \emptyset$ or $\pi_{s} \neq \emptyset$, and that Rayleigh frequencies of the compressional (shear) part are excluded if $\pi_{p}=\emptyset\left(\pi_{s}=\emptyset\right)$.

Now, our diffraction problem can be formulated as the following boundary value problem.

Direct problem (DP): Given a grating profile curve $\Lambda \subset \mathbb{R}^{2}$ (which is $2 \pi$ periodic in $x_{1}$ ) and an incident field $u^{i n}$ of the form (1) or (2), find a vector function $u=u(x ; \theta)=u^{i n}+u^{s c} \in H_{l o c}^{1}\left(\Omega_{\Lambda}\right)^{2}$ that satisfies the Navier equation (3), one of the boundary conditions (4) and (5), the quasi-periodicity ( 7$)$ and the radiation condition (10).

The inverse problem which involves the near-field measurements $u\left(x_{1}, b\right)$ for some fixed $b>\Lambda^{+}$can be formulated as follows:

Inverse problem (IP): Determine the grating profile $\Lambda$ from the knowledge of the near-field data $u\left(x_{1}, b ; \theta_{j}\right)$ for all $x_{1} \in(0,2 \pi), j=1,2, \cdots, N$. Here $u\left(x ; \theta_{j}\right)$ are solutions of (DP) corresponding to $N$ distinct incident pressure or shear waves $u^{\text {in }}$ of the form (1) or (2) with distinct incident angles $\theta_{j} \in\left(-\frac{\pi}{2}, \frac{\pi}{2}\right)(j=1,2, \cdots, N)$. (IP):

In this paper we are mainly interested in the following uniqueness questions about

Let the incident angle $\theta \in\left(-\frac{\pi}{2}, \frac{\pi}{2}\right)$ be fixed, and let $\mathcal{A}$ be the admissible class of grating profiles. Suppose the two gratings $\Lambda_{1}, \Lambda_{2} \in \mathcal{A}$ generate the total fields $u_{j}$ $(j=1,2)$ for an incident pressure resp. shear wave of the form (1) resp. (2). Does the relation

$$
u_{1}\left(x_{1}, b\right)=u_{2}\left(x_{1}, b\right), \quad \forall x_{1} \in(0,2 \pi) \text {, for some } b>\max \left\{\Lambda_{1}^{+}, \Lambda_{2}^{+}\right\}
$$

imply $\Lambda_{1}=\Lambda_{2}$ ? If not, what kind of geometric characteristics do $\Lambda_{1}$ and $\Lambda_{2}$ share in order to generate the same near field on $x_{2}=b$ ? And how many incident elastic waves are sufficient to uniquely determine an arbitrary grating profile $\Lambda \in \mathcal{A}$ ? 
3. A solvability result for (DP). In this section, we propose an equivalent variational formulation of the boundary value problem (DP) and give an existence result for the direct problem. Moreover, we construct non-trivial quasi-periodic solutions of the homogenous boundary value problem when $\Lambda$ is given by a flat grating, which provide non-uniqueness examples of (DP).

We restrict the scattering problem to a single bounded periodic cell by introducing an artificial boundary

$$
\Gamma_{b}:=\left\{\left(x_{1}, b\right): 0 \leq x_{1} \leq 2 \pi\right\}, \quad b>\Lambda^{+},
$$

and the bounded domain

$$
\Omega_{b}=\Omega_{\Lambda, b}:=\left\{\left(x_{1}, x_{2}\right) \in \Omega_{\Lambda}: 0<x_{1}<2 \pi, x_{2}<b\right\},
$$

lying between the segment $\Gamma_{b}$ and one period of the grating profile curve which we denote by $\Lambda$ again. We assume that $\Lambda$ is a Lipschitz curve, so that $\Omega_{b}$ is a bounded Lipschitz domain.

Let the energy space of our variational problem be defined by

$$
V_{\alpha}=V_{\alpha}\left(\Omega_{b}\right):=\left\{u \in H_{\alpha}^{1}\left(\Omega_{b}\right)^{2}: u \text { satisfies (4) or (5) on } \Lambda\right\}
$$

where $H_{\alpha}^{1}\left(\Omega_{b}\right)$ denotes the Sobolev space of scalar functions on $\Omega_{b}$ which are $\alpha$-quasiperiodic with respect to $x_{1}$. In the following $V_{\alpha}$ is equipped with the norm of the usual Sobolev space $H^{1}\left(\Omega_{b}\right)^{2}$ of vector functions.

Now we introduce the Dirichlet-to-Neumann (DtN) map $\mathcal{T}$ on the artificial boundary $\Gamma_{b}$. Let $H_{\alpha}^{s}\left(\Gamma_{b}\right)$ and $H_{p e r}^{s}\left(\Gamma_{b}\right)$ denote the Sobolev spaces of order $s \in \mathbb{R}$ of functions on $\Gamma_{b}$ that are $\alpha$-quasi-periodic and periodic respectively. Then, for any $u \in H_{\alpha}^{1}\left(\Omega_{b}\right)^{2}$, we have

$$
v:=\left.u\right|_{\Gamma_{b}} \in H_{\alpha}^{1 / 2}\left(\Gamma_{b}\right)^{2}, \quad \exp \left(-i \alpha x_{1}\right) v \in H_{\text {per }}^{1 / 2}\left(\Gamma_{b}\right)^{2}
$$

from the trace theorem. For any $v \in H_{\alpha}^{1 / 2}\left(\Gamma_{b}\right)^{2}$, we define $\mathcal{T} v$ as the traction $T u^{s c}$ on $\Gamma_{b}$ where $u^{s c}$ is the unique $\alpha$-quasi-periodic solution of the homogenous Navier equation in $\left\{x_{2}>b\right\}$ which satisfies (10) and $u^{s c}=v$ on $\Gamma_{b}$.

It follows from the first Betti formula that for $u, \varphi \in V_{\alpha}$

$$
-\int_{\Omega_{b}}\left(\Delta^{*}+\omega^{2}\right) u \cdot \bar{\varphi} d x=\int_{\Omega_{b}}\left(a_{L}(u, \bar{\varphi})-\omega^{2} u \cdot \bar{\varphi}\right) d x-\int_{\Gamma_{b}} \bar{\varphi} \cdot T u d s
$$

where the bar indicates the complex conjugate, and

$$
\begin{aligned}
a_{L}(u, \varphi)= & (2 \mu+\lambda)\left(\partial_{1} u_{1} \partial_{1} \varphi_{1}+\partial_{2} u_{2} \partial_{2} \varphi_{2}\right)+\mu\left(\partial_{2} u_{1} \partial_{2} \varphi_{1}+\partial_{1} u_{2} \partial_{1} \varphi_{2}\right) \\
& +\lambda\left(\partial_{1} u_{1} \partial_{2} \varphi_{2}+\partial_{2} u_{2} \partial_{1} \varphi_{1}\right)+\mu\left(\partial_{2} u_{1} \partial_{1} \varphi_{2}+\partial_{1} u_{2} \partial_{2} \varphi_{1}\right) .
\end{aligned}
$$

Making use of the relation

$$
T u=T\left(u^{s c}+u^{i n}\right)=\mathcal{T} u^{s c}+T u^{i n}=\mathcal{T} u+f_{0}, \quad \text { with } \quad f_{0}:=T u^{i n}-\mathcal{T} u^{i n},
$$

we obtain the following variational formulation of (DP): Find $u \in V_{\alpha}$ such that

$$
\int_{\Omega_{b}}\left(a_{L}(u, \bar{\varphi})-\omega^{2} u \cdot \bar{\varphi}\right) d x-\int_{\Gamma_{b}} \bar{\varphi} \cdot \mathcal{T} u d s=\int_{\Gamma_{b}} f_{0} \cdot \bar{\varphi} d s, \quad \forall \varphi \in V_{\alpha} .
$$


It should be remarked that each variational solution $u \in V_{\alpha}\left(\Omega_{b}\right)$ of (15) can be extended to a solution $u=u^{i n}+u^{s c}$ of the Navier equation (3) for $x_{2} \geq b$ via the uniqueness for the exterior Dirichlet problem (see [13]), and that each solution $u \in H_{l o c}^{1}\left(\Omega_{\Lambda}\right)^{2}$ of the boundary value problem (DP) satisfies the variational problem (15). Thus the problem (DP) and (15) are equivalent. Next we show an existence result for general incident pressure waves taking the form

$$
u_{(p)}^{i n}(x)=\sum_{\left|\alpha_{n}\right|<k_{p}} p_{n}\left(\begin{array}{c}
\alpha_{n} \\
-\beta_{n}
\end{array}\right) \exp \left[i\left(\alpha_{n} x_{1}-\beta_{n} x_{2}\right)\right]
$$

with $\alpha=k_{p} \sin \theta, p_{n} \in \mathbb{C}$, or incident shear waves taking the form

$$
u_{(s)}^{i n}(x)=\sum_{\left|\alpha_{n}\right|<k_{s}} s_{n}\left(\begin{array}{c}
\gamma_{n} \\
\alpha_{n}
\end{array}\right) \exp \left[i\left(\alpha_{n} x_{1}-\gamma_{n} x_{2}\right)\right]
$$

with $\alpha=k_{s} \sin \theta, s_{n} \in \mathbb{C}$. Note that the incident pressure wave (1) or shear wave (2) is only one term of the finite sums in (16) or (17).

THEOREM 1. Assume that the grating profile $\Lambda$ is a Lipschitz curve. Then, for all incident elastic waves of the form (16) or (17), there always exists a solution to the variational problem (15) and hence to problem (DP).

Proof. We sketch the proof, referring to [13] for more details. Let the sesquilinear form $B(u, \varphi)$ be defined by

$$
B(u, \varphi):=\int_{\Omega_{b}}\left(a_{L}(u, \bar{\varphi})-\omega^{2} u \cdot \bar{\varphi}\right) d x-\int_{\Gamma_{b}} \bar{\varphi} \cdot \mathcal{T} u d s, \forall u, \phi \in V_{\alpha},
$$

with $\mathcal{T} u:=\mathcal{T}\left(\left.u\right|_{\Gamma_{b}}\right)$. Problem (15) can be written in the form

$$
\mathcal{B} u=\mathcal{F}_{0}, \quad \mathcal{F}_{0} \in V_{\alpha}^{\prime},
$$

where $\mathcal{F}_{0}$ is given by the right hand side of (15), and the operator $\mathcal{B}: V_{\alpha} \rightarrow V_{\alpha}^{\prime}$ is defined by the sesquilinear form (18). By a detailed analysis of the DtN map $\mathcal{T}$, it is verified in [13] that $\mathcal{T}$ is the sum of a finite dimensional operator and an operator $\mathcal{T}_{1}$ satisfying

$$
\operatorname{Re}\left\{-\int_{\Gamma_{b}} \bar{u} \cdot \mathcal{T}_{1} u d s\right\} \geq 0, \quad \forall u \in H_{\alpha}^{1}\left(\Omega_{b}\right)^{2} .
$$

This together with Korn's inequality implies that the sesquilinear form $B$ is strongly elliptic over $V_{\alpha}$ and the operator $\mathcal{B}$ is always a Fredholm operator with index zero. Therefore, equation (19) is solvable if its right hand side $\mathcal{F}_{0}$ is orthogonal (with respect to the duality $(\cdot, \cdot)_{\Omega_{b}}$ extending the scalar product in $\left.L^{2}\left(\Omega_{b}\right)^{2}\right)$ to all solutions $v$ of the homogenous adjoint equation $\mathcal{B}^{*} v=0$. Note that such $v$ can always be extended to a solution of (3) in the unbounded domain $\Omega_{\Lambda}$ by setting

$$
\begin{aligned}
& v(x)= \\
& \sum_{n \in \mathbb{Z}}\left\{A_{p, n}\left(\begin{array}{c}
\alpha_{n} \\
-\bar{\beta}_{n}
\end{array}\right) \exp \left(i \alpha_{n} x_{1}-i \bar{\beta}_{n} x_{2}\right)+A_{s, n}\left(\begin{array}{c}
-\bar{\gamma}_{n} \\
-\alpha_{n}
\end{array}\right) \exp \left(i \alpha_{n} x_{1}-i \bar{\gamma}_{n} x_{2}\right)\right\},
\end{aligned}
$$

for $x_{2} \geq b$, where the Rayleigh coefficients $A_{p, n}, A_{s, n}$ are determined by the $n$-th Fourier coefficient $\hat{v}_{n}$ of $\left.e^{-i \alpha x_{1}} v\right|_{\Gamma_{b}}$ via the following relation:

$$
\hat{v}_{n}=\left(\begin{array}{cc}
\alpha_{n} & -\bar{\gamma}_{n} \\
-\bar{\beta}_{n} & -\alpha_{n}
\end{array}\right)\left(\begin{array}{c}
A_{p, n} e^{-i \bar{\beta}_{n} b} \\
A_{s, n} e^{-i \bar{\gamma}_{n} b}
\end{array}\right)
$$


On the other hand, it can be derived from

$$
\left(\mathcal{B}^{*} v, \psi\right)_{\Omega_{b}}=(v, \mathcal{B} \psi)_{\Omega_{b}}=\overline{B(\psi, v)}=0, \forall \psi \in V_{\alpha}
$$

that

$$
A_{p, n}=0 \text { for }\left|\alpha_{n}\right|<k_{p} \text { and } A_{s, n}=0 \text { for }\left|\alpha_{n}\right|<k_{s}
$$

see [13]. This means that $v$ has vanishing Rayleigh coefficients of the incoming modes, and therefore,

$$
\hat{v}_{n}=(0,0)^{\top} \text { for }\left|\alpha_{n}\right| \leq k_{p}<k_{s}, \quad \hat{v}_{n}=\left(\alpha_{n},-\bar{\beta}_{n}\right)^{\top} A_{p, n} \exp \left(-i \bar{\beta}_{n} b\right) \text { for }\left|\alpha_{n}\right| \leq k_{s} .
$$

Through direct calculations, we deduce that $f_{0}:=T u^{i n}-\mathcal{T} u^{i n}$ takes the form

$$
f_{0}=\sum_{\left|\alpha_{n}\right|<k_{p}} p_{n} \frac{2 i \beta_{n} k_{p}(\lambda+2 \mu)}{\alpha_{n}^{2}+\beta_{n} \gamma_{n}}\left(\begin{array}{c}
-\alpha_{n} \\
\gamma_{n}
\end{array}\right) e^{-i \beta_{n} b} e^{i \alpha_{n} x_{1}}=: \sum_{\left|\alpha_{n}\right|<k_{p}} h_{n} e^{i \alpha_{n} x_{1}}
$$

for the incident pressure wave $u_{(p)}^{i n}$ defined in (16), which leads to

$$
\mathcal{F}_{0}(v)=\int_{\Gamma_{b}} f_{0} \cdot \bar{v} d s=2 \pi \sum_{\left|\alpha_{n}\right|<k_{p}} h_{n} \cdot \overline{\hat{v}_{n}}=0
$$

For the incident shear wave $u_{(s)}^{i n}$ defined in (17), we obtain

$$
f_{0}=\sum_{\left|\alpha_{n}\right|<k_{s}} s_{n} \frac{-2 i \gamma_{n} k_{s} \mu}{\alpha_{n}^{2}+\beta_{n} \gamma_{n}}\left(\begin{array}{c}
\beta_{n} \\
\alpha_{n}
\end{array}\right) e^{-i \gamma_{n} b} e^{i \alpha_{n} x_{1}}:=\sum_{\left|\alpha_{n}\right|<k_{s}} g_{n} e^{i \alpha_{n} x_{1}}
$$

so that

$$
\begin{aligned}
\mathcal{F}_{0}(v)=\int_{\Gamma_{b}} f_{0} \cdot \bar{v} d s & =2 \pi \sum_{\left|\alpha_{n}\right|<k_{s}} g_{n} \cdot \overline{\hat{v}_{n}} \\
& =2 \pi \sum_{\left|\alpha_{n}\right|<k_{s}} s_{n} e^{-i \gamma_{n} b} \frac{-2 i \gamma_{n} k_{s} \mu}{\alpha_{n}^{2}+\beta_{n} \gamma_{n}}\left(\begin{array}{ll}
\beta_{n} & \alpha_{n}
\end{array}\right) \cdot \bar{A}_{p, n} e^{i \beta_{n} b}\left(\begin{array}{c}
\alpha_{n} \\
-\beta_{n}
\end{array}\right) \\
& =0 .
\end{aligned}
$$

Therefore, the right hand side of equation (19) is always orthogonal to each solution of (22). Applying the Fredholm alternative completes the proof.

It is shown in [13] that uniqueness holds for the Dirichlet problem if $\Lambda$ is given by the graph of a Lipschitz function. However, the uniqueness for the third or fourth kind boundary value problem is not valid even if $\Lambda$ can be represented by a smooth function. This can be seen from the scattering problem for flat gratings; see the following theorem.

TheOrem 2. Assume $\Lambda:=\left\{x_{2}=a\right\}$, and let $u$ satisfy the Rayleigh expansion (10) in $x_{2}>a$.

(i) If $u$ satisfies the boundary conditions of the third kind (4) on $\Lambda$, then

$$
u=c_{1} e_{1} \exp \left(i k_{p} x_{1}\right)+c_{2} e_{1} \exp \left(-i k_{p} x_{1}\right) .
$$


(ii) If u satisfies the boundary conditions of the fourth kind (5) on $\Lambda$, then

$$
u=c_{3} e_{2} \exp \left(i k_{s} x_{1}\right)+c_{4} e_{2} \exp \left(-i k_{s} x_{1}\right) .
$$

Here $c_{j} \in \mathbb{C}(j=1,2,3,4)$ are constants, $e_{1}=(1,0)^{\top}$ and $e_{2}=(0,1)^{\top}$.

Proof. We assume the boundary conditions of the third kind, $\mathbf{n} \cdot u=\tau \cdot T u=0$, are imposed on $\Lambda$. Since $\mathbf{n}=(0,1)^{\top}, \tau=(-1,0)^{\top}$ on $\left\{x_{2}=a\right\}$, these boundary conditions take the form

$$
u_{2}=0 \text { and } \partial_{2} u_{1}=0 \text { on } \Lambda .
$$

Inserting the Rayleigh expansion (10) into the conditions (24) and using the fact that $\left\{\exp \left(i \alpha_{n} x_{1}\right), n \in \mathbb{Z}\right\}$ is an orthogonal basis of $L^{2}(0,2 \pi)$, we obtain

$$
\left(\begin{array}{cc}
\beta_{n} & -\alpha_{n} \\
i \alpha_{n} \beta_{n} & i \gamma_{n}^{2}
\end{array}\right)\left(\begin{array}{c}
A_{p, n} e^{i \beta_{n} a} \\
A_{s, n} e^{i \gamma_{n} a}
\end{array}\right)=0 \quad \text { for } n \in \mathbb{Z} .
$$

Since $\gamma_{n}^{2}+\alpha_{n}^{2}=k_{s}^{2} \neq 0, \forall n \in \mathbb{Z}$, it follows from $(25)$ that $A_{s, n}=A_{p, n}=0$ if $\beta_{n} \neq 0$, and $A_{s, n}=0$ if $\beta_{n}=0$. In view of the definition of $\beta_{n}$ (see (11)), we can complete the proof in the case of the third kind boundary conditions. Noting that the boundary conditions of the fourth kind on $\left\{x_{2}=a\right\}$ can be written as $u_{1}=0$ and $\partial_{2} u_{2}=0$ on $\Lambda$, we can prove the second assertion analogously.

REMARK 1. If $\Lambda:=\left\{x_{2}=a\right\}$, then the problem (DP) admits a unique solution if Rayleigh frequencies of the compressional (resp. shear) part are excluded under the third (resp. fourth) kind boundary conditions.

4. Inverse scattering by flat gratings. We begin with introducing some notations that will be used throughout the following sections.

1. $A^{\#}$ : the number of elements in a set $A$.

2. $\left|A_{1} A_{2}\right|$ : the length of a line segment $A_{1} A_{2}$ with end points $A_{1}, A_{2} \in \mathbb{R}^{2}$.

3. $|c|$ : the modulus of a number $c \in \mathbb{C} ;\|x\|$ : the Euclidean norm of a vector $x \in \mathbb{R}^{2}$.

4. $\mathrm{R}_{l}$ : the reflection with respect to a line $l$ in $\mathbb{R}^{2}$.

5. $\mathrm{R}_{l}^{\prime}$ : the reflection with respect to the line $l^{\prime}$ that passes through the origin $O$ and is parallel to $l$.

TheOREM 3. Let $\Lambda_{j}:=\left\{x_{2}=b_{j}\right\}$ where $b_{j}$ is a constant satisfying $\left|b_{j}\right|<b$ $(j=1,2)$, and assume that $u_{j}:=u_{j}(x ; \theta)$ satisfies problem (DP) corresponding to the profile $\Lambda_{j}$. If

$$
u_{1}\left(x ; \theta_{m}\right)=u_{2}\left(x ; \theta_{m}\right) \quad \text { on } \quad x_{2}=b
$$

holds for $2 \mathcal{B}^{\#}+1$ incident pressure (shear) waves of the form (1)((2)) with distinct incident angles $\theta_{m} \in\left(-\frac{\pi}{2}, \frac{\pi}{2}\right)\left(m=1,2, \cdots, 2 \mathcal{B}^{\#}+1\right)$, then $b_{1}=b_{2}$. Here

$$
\mathcal{B}:=\left\{n \in \mathbb{Z}:|n|<\frac{2 b k}{\pi}\right\}
$$

with $k=k_{p}$ for an incident pressure wave or $k=k_{s}$ for an incident shear wave.

Proof. Suppose the total fields $u_{j}$ satisfy the boundary conditions of the fourth kind on $\Lambda_{j}(j=1,2)$, and assume $u^{i n}$ is the incident pressure wave defined in (1). We 
shall prove the theorem by contradiction. Assume that $b_{1} \neq b_{2}$. Following the proof of Theorem 2, we can derive that

$$
u_{j}(x)=\hat{\theta} e^{i k_{p} x \cdot \hat{\theta}}-\left(\begin{array}{c}
\sin \theta \\
\cos \theta
\end{array}\right) e^{i\left(\alpha x_{1}+\beta\left(x_{2}-2 b_{j}\right)\right.}+\sum_{\gamma_{n}=0} A_{s, n}^{(j)}\left(\begin{array}{c}
0 \\
-\alpha_{n}
\end{array}\right) e^{i \alpha_{n} x_{1}}, \quad \text { in } x_{2}>b_{j}
$$

for $j=1,2$. Substituting (28) into (26) gives

$$
e^{i \beta\left(b-2 b_{1}\right)}=e^{i \beta\left(b-2 b_{2}\right)}, \text { and } A_{s, n}^{(1)}=A_{s, n}^{(2)} \text { if } \gamma_{n}=0,
$$

which implies the relation

$$
b_{2}-b_{1}=\frac{\pi}{\beta} m=\frac{\pi}{k_{p} \cos \theta} m, \text { for some } m \in \mathbb{Z} .
$$

Since $b_{2}-b_{1}<2 b, m$ must belong to the set $\mathcal{B}$ defined in (27) with $k=k_{p}$. Thus, it is clear from (26) that for each incident angle $\theta_{m}$, there exists some $n_{m} \in \mathcal{B}$. Moreover $n_{m_{1}}=n_{m_{2}}$ if and only if $\theta_{m_{1}}=\theta_{m_{2}}$ or $\theta_{m_{1}}=-\theta_{m_{2}}$. Therefore, if (26) holds for $2 \mathcal{B}^{\#}+1$ incident waves with distinct incident angles, then $\mathcal{B}$ contains at least $\mathcal{B}^{\#}+1$ elements, which is impossible. The other cases can be proved by a similar argument. $\square$

REMARK 2. It follows from $\mathcal{B}^{\#} \rightarrow \infty$ as $k \rightarrow \infty$ or $b \rightarrow \infty$ that a fixed number of incident elastic waves is not sufficient to uniquely determine an arbitrary flat grating, since the corresponding counterexamples can be readily constructed from the proof of Theorem 3. In fact, if the number of incident pressure waves is $N \in \mathbb{N}$, then in the case of the fourth kind boundary conditions, we may choose the grating profiles $\Lambda_{1}=\left\{x_{2}=0\right\}, \Lambda_{2}=\left\{x_{2}=\pi\right\}$, take $k_{p}>N$, and let $k_{s}$ and the incident angles $\theta_{j}$ $(j=1,2 \cdots, N)$ satisfy

$$
\theta_{j}>0, \quad \cos \theta_{j}=\frac{j}{k_{p}}, \gamma_{n}\left(\theta_{j}\right) \neq 0, \quad \text { for } j=1,2 \cdots, N, \quad \forall n \in \mathbb{Z} .
$$

It follows from (28) that the total fields $u_{j}^{(m)}(x)$ corresponding to $\theta_{j}(j=1,2 \cdots, N)$, $\Lambda_{m}(m=1,2)$ can be written as

$$
\begin{aligned}
& u_{j}^{(1)}(x)=\left(\begin{array}{c}
\sin \theta_{j} \\
-\cos \theta_{j}
\end{array}\right) e^{i k_{p}\left(x_{1} \sin \theta_{j}-x_{2} \cos \theta_{j}\right)}-\left(\begin{array}{c}
\sin \theta_{j} \\
\cos \theta_{j}
\end{array}\right) e^{i k_{p}\left(x_{1} \sin \theta_{j}+x_{2} \cos \theta_{j}\right)}, \\
& u_{j}^{(2)}(x)=\left(\begin{array}{c}
\sin \theta_{j} \\
-\cos \theta_{j}
\end{array}\right) e^{i k_{p}\left(x_{1} \sin \theta_{j}-x_{2} \cos \theta_{j}\right)}-\left(\begin{array}{c}
\sin \theta_{j} \\
\cos \theta_{j}
\end{array}\right) e^{i k_{p}\left(x_{1} \sin \theta_{j}+\left(x_{2}-2 \pi\right) \cos \theta_{j}\right)} .
\end{aligned}
$$

Moreover, it can be verified from $k_{p} \cos \theta_{j} \in \mathbb{N}$ that

$$
u_{j}^{(1)}\left(x_{1}, b ; \theta_{j}\right)=u_{j}^{(2)}\left(x_{1}, b ; \theta_{j}\right), \quad \forall b>\pi, j=1,2 \cdots, N .
$$

Thus $N$ incident plane pressure waves are not enough to uniquely determine a flat grating under the fourth kind boundary conditions. The counterexamples for the other cases can be constructed similarly. This implies that the global uniqueness by finitely many incoming plane elastic waves is impossible for general periodic gratings.

To prove global uniqueness for the inverse problem, we therefore exclude flat gratings by defining the following admissible class of periodic grating profiles:

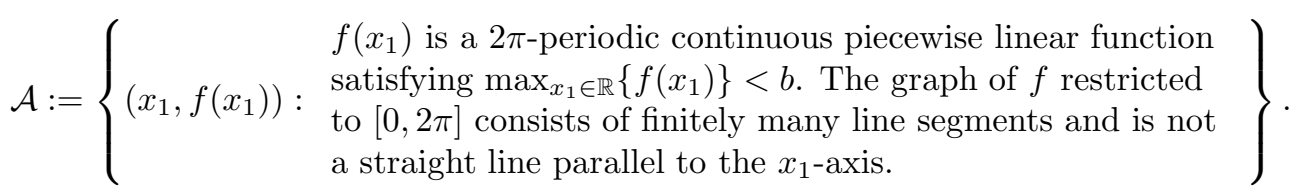


5. Auxiliary lemmas. The following two lemmas play an important role in this paper; the first one can be found in [9], while the second one on the reflection principle for the Navier equation is the main tool of this paper.

Lemma 1. Let $a_{j} \in \mathbb{C}^{2}$, and let $\lambda_{j} \in \mathbb{R}$ be distinct numbers $(j=1,2, \cdots, n)$. If

$$
\sum_{j=1}^{n} a_{j} \exp \left(i \lambda_{j} t\right)=0, \quad \forall t \in \mathbb{R}
$$

then $a_{j}=(0,0)^{\top}, j=1,2, \cdots, n$.

Definition 1. Let $S \subset \Omega_{\Lambda}$ be a ray starting from one point and leading to infinity in $\left\{x_{2}>b\right\}$ for $b>\Lambda^{+}$. If $u$ satisfies the boundary conditions of the third (fourth) kind on $S$, then $S$ is called a third (fourth) kind ray of u. Similarly, $S$ is called a third (fourth) kind line of $u$ if $S$ is a straight line on which $u$ satisfies the corresponding conditions.

Lemma 2 (Reflection principle for the Navier equation). Let $\Omega$ be a symmetric domain with respect to a line $l$, and let $\tilde{l} \subset \Omega$ be a subset of another line such that $\mathrm{R}_{l}(\tilde{l}) \subset \Omega$. Assume $u \in H^{1}(\Omega)^{2}$ satisfies the Navier equation $\triangle^{*} u+\omega^{2} u=0$ in $\Omega$.

(i) If $u$ satisfies the boundary conditions of the fourth kind, $\tau \cdot u=n \cdot T u=0$ on $l \cap \Omega$, then

$$
u(x)+\mathrm{R}_{l}^{\prime}\left(u\left(\mathrm{R}_{l}(x)\right)\right)=0 \quad \text { in } \Omega .
$$

(ii) If $u$ satisfies the boundary conditions of the third kind, $n \cdot u=\tau \cdot T u=0$ on $l \cap \Omega$, then

$$
u(x)-\mathrm{R}_{l}^{\prime}\left(u\left(\mathrm{R}_{l}(x)\right)\right)=0 \quad \text { in } \Omega .
$$

In particular, if $\tilde{l}$ is a fourth (third) kind line of $u$ in the case $(i)((i i))$, then $\mathrm{R}_{l}(\tilde{l})$ is also a fourth (third) kind line of $u$.

REMARK 3. The original version of the reflection principle for the Navier equation can be found in [17], which is proved in the three dimensional case when the domain $\Omega$ is symmetric with respect to the $x_{1} x_{2}$-plane. The proof readily carries over to the two dimensional domain $\Omega$ in the above lemma. Note that the relations (29) and (30) are similar to those given in [21, 22] for the Maxwell equations.

In the following, we denote by $u_{j}(x ; \theta)$ the corresponding total fields for (DP) associated with the profiles $\Lambda_{j}:=\left\{x_{2}=f_{j}\left(x_{1}\right)\right\} \in \mathcal{A}(j=1,2)$, and assume $b>$ $\max \left\{\Lambda_{1}^{+}, \Lambda_{2}^{+}\right\}$.

Lemma 3. If the relation $u_{1}\left(x_{1}, b ; \theta\right)=u_{2}\left(x_{1}, b ; \theta\right), \forall x_{1} \in(0,2 \pi)$, holds for two different grating profiles $\Lambda_{1}, \Lambda_{2} \in \mathcal{A}$, then

(i) Under the boundary conditions of the third (fourth) kind, there always exists a third (fourth) kind ray of both $u_{1}$ and $u_{2}$. Moreover, this ray is non-parallel to the coordinate axes.

(ii) Both the total fields $u_{j}=u^{i n}+u_{j}^{s c}, j=1,2$, can be reduced to a finite sum of propagating waves, i.e.,

$$
\begin{aligned}
u_{1}=u_{2}=u^{i n} & +\sum_{\left|\alpha_{n}\right| \leq k_{p}} A_{p, n}\left(\begin{array}{c}
\alpha_{n} \\
\beta_{n}
\end{array}\right) e^{i\left(\alpha_{n} x_{1}+\beta_{n} x_{2}\right)} \\
& +\sum_{\left|\alpha_{n}\right| \leq k_{s}} A_{s, n}\left(\begin{array}{c}
\gamma_{n} \\
-\alpha_{n}
\end{array}\right) e^{i\left(\alpha_{n} x_{1}+\gamma_{n} x_{2}\right)}
\end{aligned}
$$


for $x_{2}>\max \left\{\Lambda_{1}^{+}, \Lambda_{2}^{+}\right\}$.

Proof. (i) Since $\Lambda_{j}$ is piecewise linear and $u_{j} \in H_{l o c}^{1}\left(\Omega_{\Lambda_{j}}\right)^{2}$, from the standard elliptic regularity theory it follows that $u_{j}$ is infinitely smooth up to $\Lambda_{j}$ except for the corner points and is real-analytic in $\Omega_{\Lambda_{j}}$. It follows from the assumption $u_{1}\left(x_{1}, b ; \theta\right)=$ $u_{2}\left(x_{1}, b ; \theta\right), \forall x_{1} \in(0,2 \pi)$, and the uniqueness of the Dirichlet problem (see $\left.[3,13]\right)$ that $u_{1}=u_{2}$ for $x_{2} \geq b$. By the unique continuation of solutions to the Navier equation, we have

$$
u_{1}=u_{2} \text { for } x_{2}>\max \left\{\Lambda_{1}^{+}, \Lambda_{2}^{+}\right\} .
$$

Relying on the analyticity of $u_{j}$ and the reflection principle for the Navier equation, the 'exit' ray mentioned in assertion (i) can always be found if $\Lambda_{1} \neq \Lambda_{2}$ and $\Lambda_{j}$ $(j=1,2)$ are given by the graphs of piecewise linear functions. We refer to [15] for the details in the case of the Helmholtz equation with the Dirichlet or Neumann boundary condition, and the argument can be carried over to the Navier equation with the boundary conditions of the third or fourth kind.

(ii) Following [15], we prove the second assertion under the fourth kind boundary conditions. The proof under the third kind boundary conditions is analogous. By the identity (32), we can write

$$
u(x)=u_{1}(x)=u_{2}(x)=I(x)+\sum_{\left|\alpha_{n}\right|>k_{p}} I_{p, n}(x)+\sum_{\left|\alpha_{n}\right|>k_{s}} I_{s, n}(x)
$$

for all $x_{1} \in \mathbb{R}$ and $x_{2}>\max \left\{\Lambda_{1}^{+}, \Lambda_{2}^{+}\right\}$, where

$$
\begin{aligned}
I_{p, n}\left(x_{1}, x_{2}\right) & :=A_{p, n}\left(\alpha_{n}, \beta_{n}\right)^{\top} e^{i\left(\alpha_{n} x_{1}+\beta_{n} x_{2}\right)}, I_{s, n}\left(x_{1}, x_{2}\right):=A_{s, n}\left(-\gamma_{n}, \alpha_{n}\right)^{\top} e^{i\left(\alpha_{n} x_{1}+\gamma_{n} x_{2}\right)}, \\
I\left(x_{1}, x_{2}\right) & :=u^{i n}\left(x_{1}, x_{2}\right)+\sum_{\left|\alpha_{n}\right| \leq k_{p}} I_{p, n}\left(x_{1}, x_{2}\right)+\sum_{\left|\alpha_{n}\right| \leq k_{s}} I_{s, n}\left(x_{1}, x_{2}\right) .
\end{aligned}
$$

Without loss of generality, we let $l:=\left\{x_{2}=k x_{1}: x_{1}>0\right\}$, for some $k \neq 0$, be the fourth kind ray involved in assertion $(i)$, on which $u$ satisfies $\tau \cdot u=\mathbf{n} \cdot T u=0$ with $\tau=\frac{1}{\sqrt{1+k^{2}}}(1, k)$. We then have

$$
\begin{aligned}
0=\left.\tau \cdot u\right|_{l}=\tau \cdot I\left(x_{1}, k x_{1}\right) & +\sum_{\left|\alpha_{n}\right|>k_{p}} \tau \cdot I_{p, n}\left(x_{1}, k x_{1}\right) \\
& +\sum_{\left|\alpha_{n}\right|>k_{s}} \tau \cdot I_{s, n}\left(x_{1}, k x_{1}\right), \forall x_{1}>0 .
\end{aligned}
$$

Noting that $\tau \cdot I\left(x_{1}, k x_{1}\right)$ is an almost periodic function on $\mathbb{R}^{+}$, and $\tau \cdot I_{p, n}\left(x_{1}, k x_{1}\right)$ for $\left|\alpha_{n}\right|>k_{p}, \tau \cdot I_{s, n}\left(x_{1}, k x_{1}\right)$ for $\left|\alpha_{n}\right|>k_{s}$ are exponentially decaying functions as $x_{1} \rightarrow+\infty$, we obtain from (33) that (see, e.g., [12, P. 407] concerning the first equality)

$$
\begin{aligned}
\max _{x_{1} \in \mathbb{R}^{+}}\left|\tau \cdot I\left(x_{1}, k x_{1}\right)\right| & =\limsup _{x_{1} \rightarrow+\infty}\left|\tau \cdot I\left(x_{1}, k x_{1}\right)\right| \\
& =\limsup _{x_{1} \rightarrow+\infty}\left\{\sum_{\left|\alpha_{n}\right|>k_{p}} \tau \cdot I_{p, n}\left(x_{1}, k x_{1}\right)+\sum_{\left|\alpha_{n}\right|>k_{s}} \tau \cdot I_{s, n}\left(x_{1}, k x_{1}\right)\right\} \\
& \leq \epsilon,
\end{aligned}
$$


for any $\epsilon>0$. Thus $\tau \cdot I\left(x_{1}, k x_{1}\right) \equiv 0$. This together with (33) implies that

$$
\begin{aligned}
\sum_{\left|\alpha_{n}\right|>k_{p}} A_{p, n}\left(\alpha_{n}+i k\left|\beta_{n}\right|\right) e^{i \alpha_{n} x_{1}-\left|\beta_{n}\right| k x_{1}} & \\
& +\sum_{\left|\alpha_{n}\right|>k_{s}} A_{s, n}\left(-i\left|\gamma_{n}\right|+k \alpha_{n}\right) e^{i \alpha_{n} x_{1}-\left|\gamma_{n}\right| k x_{1}}=0
\end{aligned}
$$

for all $x_{1}>0$. Let $A^{*}=\min \left\{\inf _{\left|\alpha_{n}\right|>k_{p}}\left\{\left|\beta_{n}\right|\right\}, \inf _{\left|\alpha_{n}\right|>k_{s}}\left\{\left|\gamma_{n}\right|\right\}\right\}$. Then there exist at most four different indices $n_{1}, n_{2}, m_{1}, m_{2} \in \mathbb{Z}$ such that

$$
\beta_{n_{1}}=\beta_{n_{2}}=\gamma_{m_{1}}=\gamma_{m_{2}}=i A^{*}, \quad \alpha_{n_{1}}=-\alpha_{n_{2}}>k_{p}, \alpha_{m_{1}}=-\alpha_{m_{2}}>k_{s} .
$$

Multiplying (34) by $\exp \left(A^{*} k x_{1}\right)$ and letting $x_{1} \rightarrow+\infty$, we obtain

$$
\begin{aligned}
0= & A_{p, n_{1}}\left(\alpha_{n_{1}}+i k A^{*}\right) \exp \left(i \alpha_{n_{1}} x_{1}\right)+A_{p, n_{2}}\left(-\alpha_{n_{1}}+i k A^{*}\right) \exp \left(-i \alpha_{n_{1}} x_{1}\right) \\
& +A_{s, m_{1}}\left(-i A^{*}+k \alpha_{m_{1}}\right) \exp \left(i \alpha_{m_{1}} x_{1}\right)+A_{s, m_{2}}\left(-i A^{*}-k \alpha_{m_{1}}\right) \exp \left(-i \alpha_{m_{1}} x_{1}\right)
\end{aligned}
$$

for $x_{1}>0$. Since $\pm \alpha_{n_{1}}, \pm \alpha_{m_{1}}$ are four different real numbers and $k$ is non-zero, we obtain from Lemma 1 that

$$
A_{p, n_{1}}=A_{p, n_{2}}=A_{s, m_{1}}=A_{s, m_{2}}=0 .
$$

Setting $A^{* *}=\min \left\{\inf _{\left|\beta_{n}\right|>A^{*}}\left\{\left|\beta_{n}\right|\right\}, \inf _{\left|\gamma_{n}\right|>A^{*}}\left\{\left|\gamma_{n}\right|\right\}\right\}$ and repeating the argument above, we see that $A_{p, n}=0$ for $\left|\alpha_{n}\right|>k_{p}$ and $A_{s, n}=0$ for $\left|\alpha_{n}\right|>k_{s}$.

6. Inverse scattering of incident pressure waves. In this section, the uniqueness of inverse scattering of incident pressure waves by one-dimensional diffraction gratings is investigated. Throughout this section, we make the following assumptions:

(A1) The incident wave is the incident pressure wave defined in (1), i.e. $u^{i n}:=$ $\hat{\theta} \exp \left(i k_{p} x \cdot \hat{\theta}\right)$ with some fixed incident angle $\theta \in\left(-\frac{\pi}{2}, \frac{\pi}{2}\right)$.

(A2) $\Lambda_{1}, \Lambda_{2} \in \mathcal{A}$ are two different grating profiles. Furthermore, without loss of generality we suppose that one of the profiles $\Lambda_{1}, \Lambda_{2}$ has a corner point at the origin $O=(0,0)$.

(A3) $u_{1}(x ; \theta)=u_{2}(x ; \theta)$ holds on $\Gamma_{b}$.

By Lemma $3(i i)$, we have $u=u_{1}=u_{2}$ on $\mathbb{R}^{2}$. Let $\Lambda$ denote one of the profiles $\Lambda_{j}(j=1,2)$, and define $(\alpha, \beta):=\left(\alpha_{0}, \beta_{0}\right)=\left(k_{p} \sin \theta, k_{p} \cos \theta\right)$.

6.1. Rotational invariance. In this subsection, based on the reflection principle for the Navier equation, we will prove that the total field $u$ of (31) remains rotationally invariant, and then use such invariance to determine the finite number of propagating directions and Rayleigh coefficients of the compressional part, whereas the shear part of the total field is proved to be empty.

Since both the normal vector $\mathbf{n}$ and the tangential vector $\tau$ on a straight line are constant vectors, and since by Lemma $3(i i) u$ is an analytic function in $\mathbb{R}^{2}$, each line segment of $\Lambda$ can be extended to a third (fourth) kind line of $u$ in $\mathbb{R}^{2}$. Thus, by the definition of $\mathcal{A}$, there exist at least two third (fourth) kind lines, say $L_{1}$ and $L_{2}$, extending two line segments of $\Lambda$. Furthermore, both $L_{1}$ and $L_{2}$ are not parallel to the $x_{2}$-axis since each element of $\mathcal{A}$ is given by the graph of a function. By assumption (A2), we may suppose $L_{1} \cap L_{2}=O$, and then $u$ takes the form

$$
u=\sum_{n \in P} A_{p, n} \mathbf{P}_{n} \exp \left(i x \cdot \mathbf{P}_{n}\right)+\sum_{n \in S} A_{s, n} \mathbf{S}_{n}^{\perp} \exp \left(i x \cdot \mathbf{S}_{n}\right) \quad \text { in } \mathbb{R}^{2},
$$

where 


$$
\begin{aligned}
& P:=\left\{n \in \mathbb{Z}:\left|\alpha_{n}\right| \leq k_{p}, A_{p, n} \neq 0\right\} \cup\{\kappa\}, S:=\left\{n \in \mathbb{Z}:\left|\alpha_{n}\right| \leq k_{s}, A_{s, n} \neq 0\right\} \\
& \mathbf{P}_{n}=\left(\alpha_{n}, \beta_{n}\right)^{\top} \text { for } n \in P \backslash\{\kappa\}, \mathbf{P}_{\kappa}=(\alpha,-\beta)^{\top}, \mathbf{S}_{n}=\left(\alpha_{n}, \gamma_{n}\right)^{\top} \text { for } n \in S, A_{p, \kappa}=\frac{1}{k_{p}} .
\end{aligned}
$$

For convenience we introduce the following notations:

$$
\mathcal{P}=\left\{\mathbf{P}_{n}: n \in P\right\}, \quad \mathcal{S}=\left\{\mathbf{S}_{n}: n \in S\right\} .
$$

Note that $\mathcal{P}$ consists of a finite number of upward propagating directions of the compressional part of $u,\left\{\mathbf{P}_{n}: n \in P \backslash\{\kappa\}\right\}$, as well as the incident direction $\mathbf{P}_{\kappa}$, whereas $\mathcal{S}$ consists of finitely many upward propagating directions of the shear part of $u$.

REMARK 4. (i) By the definitions of $\alpha_{n}, \beta_{n}$ and $\gamma_{n}$ (see (11)), we have that $\mathcal{P} \subset B_{k_{p}}(O)$ and $\mathcal{S} \subset B_{k_{s}}(O)$, where $B_{r}(O):=\left\{x \in \mathbb{R}^{2}:\|x\|=r\right\}$ denotes the circle centered at the origin $O$ with radius $r$.

(ii) Since the plane pressure wave of the form (1) is taken as an incident wave, $\boldsymbol{P}_{\kappa}$ is the unique element in $\mathcal{P}$ whose $x_{2}$-component is negative. Moreover, if $\pi_{p}=\emptyset$, then each element of $\mathcal{P}$ but $\boldsymbol{P}_{\kappa}$ has a positive $x_{2}$-component; and if $\pi_{p} \neq \emptyset$, at most two elements of $\mathcal{P},\left(k_{p}, 0\right)$ and $\left(-k_{p}, 0\right)$, have vanishing $x_{2}$-components. The $x_{2}$ components of the elements in $\mathcal{S}$ are all positive if $\pi_{s}=\emptyset$, while $\left(k_{s}, 0\right)$ or $\left(-k_{s}, 0\right)$ belongs to $\mathcal{S}$ if $\pi_{s} \neq \emptyset$. Recall that $\pi_{p}$ and $\pi_{s}$ are defined in (12).

By the $\alpha$-quasi-periodicity of solutions of (DP), we arrive at

Lemma 4. If $(-\alpha, \beta) \in \mathcal{P}$, then $2 k_{p} \sin \theta \in \mathbb{Z}$. If $\left( \pm k_{p}, 0\right) \in \mathcal{P}$, then $k_{p}(1 \mp$ $\sin \theta) \in \mathbb{Z}$. Finally, if $\left\{(-\alpha, \beta),\left(k_{p}, 0\right),\left(-k_{p}, 0\right)\right\} \subset \mathcal{P}$, then $k_{p}(1+\sin \theta) \in \mathbb{Z}$ and $k_{p}(1-\sin \theta) \in \mathbb{Z}$

Define

$$
D=\left\{\begin{array}{l}
l \text { is a line that passes through the origin } O \text {. Furthermore, } l \text { is a } \\
l: \quad \text { third (fourth) kind line of } u \text { corresponding to the boundary } \\
\text { conditions of the third (fourth) kind on the grating profile. }
\end{array}\right\} .
$$

Obviously, we have $D^{\#} \geq 2$ since $L_{1}, L_{2} \in D$. Next we derive some important properties of $D$ by the reflection principle. The following lemma tells us that $D^{\#}<\infty$ and the angles formed by every two neighboring lines of $D$ are all equal.

LEMMA 5. D consists of a finite number of lines which form an equi-angular system of lines in $\mathbb{R}^{2}$.

Proof. We refer to [9] or [14] for the proof in the case of the Maxwell and Helmholtz equations. Note that the result is already implicitly contained in [15] and [16]. $\mathrm{\square}$

For all $l \in D$, the reflection $\mathrm{R}_{l}$ can be represented via an orthogonal matrix such that $\mathrm{R}_{l}(x) \cdot y=x \cdot \mathrm{R}_{l}(y)$. Thus, it follows from the reflection principle that

$$
\begin{aligned}
0= & \sum_{n \in P} A_{p, n}\left[\mathbf{P}_{n} \exp \left(i x \cdot \mathbf{P}_{n}\right) \pm \mathrm{R}_{l}\left(\mathbf{P}_{n}\right) \exp \left(i x \cdot \mathrm{R}_{l}\left(\mathbf{P}_{n}\right)\right)\right] \\
& +\sum_{n \in S} A_{s, n}\left[\mathbf{S}_{n}^{\perp} \exp \left(i x \cdot \mathbf{S}_{n}\right) \pm \mathrm{R}_{l}\left(\mathbf{S}_{n}^{\perp}\right) \exp \left(i x \cdot \mathrm{R}_{l}\left(\mathbf{S}_{n}\right)\right)\right]
\end{aligned}
$$

holds in $\mathbb{R}^{2}$, where + or - is taken for the fourth or third kind boundary conditions, respectively. In view of Lemma 1 , the following lemma can be readily derived from the above identity. 
Lemma 6. Assume $l \in D$.

(i) $\mathrm{R}_{l}(\mathcal{P})=\mathcal{P}, \mathrm{R}_{l}(\mathcal{S})=\mathcal{S}$.

(ii) If $\mathrm{R}_{l}\left(\boldsymbol{P}_{n}\right)=\boldsymbol{P}_{m}$ for $n, m \in P$, then

$$
A_{p, n}+A_{p, m}=0 \quad \text { resp. } \quad A_{p, n}-A_{p, m}=0
$$

under the boundary conditions of the fourth resp. third kind. If $\mathrm{R}_{l}\left(\boldsymbol{S}_{n}\right)=\boldsymbol{S}_{m}$ for $n, m \in S$, then

$$
A_{s, n} \mathrm{R}_{l}\left(\boldsymbol{S}_{n}^{\perp}\right)+A_{s, m} \boldsymbol{S}_{m}^{\perp}=0 \quad \text { resp. } \quad A_{s, n} \mathrm{R}_{l}\left(\boldsymbol{S}_{n}^{\perp}\right)-A_{s, m} \boldsymbol{S}_{m}^{\perp}=0
$$

under the boundary conditions of the fourth resp. third kind.

(iii) In the case of the fourth kind boundary conditions, $\mathrm{R}_{l}\left(\boldsymbol{P}_{n}\right) \neq \boldsymbol{P}_{n}$ for all $n \in P$, and in particular, $\mathbf{R}_{l}\left(\boldsymbol{P}_{\kappa}\right) \neq \boldsymbol{P}_{\kappa}$ for the incident direction $\boldsymbol{P}_{\kappa}=(\alpha,-\beta)^{\top}$. In the case of the third kind boundary conditions, $\mathrm{R}_{l}\left(\boldsymbol{S}_{n}\right) \neq \boldsymbol{S}_{n}$ for all $n \in S$.

Let $\operatorname{Rot}_{\varphi}$ be the rotation around the origin $O$ by the angle $\varphi$, and let $\operatorname{Ref}_{\varphi}$ be the reflection about the line $L$ through the origin which makes an angle $\varphi$ with the positive $x_{1}$-axis. Both $\operatorname{Rot}_{\varphi}$ and $\operatorname{Ref}_{\varphi}$ can be represented via orthogonal matrices. With these definitions and representations, the following identity can be justified:

$$
\operatorname{Ref}_{\varphi_{1}} \operatorname{Ref}_{\varphi_{2}}=\operatorname{Rot}_{2\left(\varphi_{1}-\varphi_{2}\right)} .
$$

Lemma 7 (Rotational invariance). We have that

$$
\operatorname{Rot}_{\frac{2 \pi}{D \#}}(u(x))=u\left(\operatorname{Rot}_{\frac{2 \pi}{D \#}}(x)\right), \operatorname{Rot}_{\frac{2 \pi}{D \#}}(\mathcal{P})=\mathcal{P}, \operatorname{Rot}_{\frac{2 \pi}{D \#}}(\mathcal{S})=\mathcal{S} .
$$

Proof. It follows from Lemma 5 that the angle formed by every two neighboring lines of $D$ is $\frac{\pi}{D^{\#}}$. Thus, by (36) we may choose two neighboring lines of $D, l_{1}$ and $l_{2}$, such that

$$
\operatorname{Rot}_{\frac{2 \pi}{D \#}}(x)=\mathrm{R}_{l_{1}} \mathrm{R}_{l_{2}}(x) \quad \forall x \in \mathbb{R}^{2} .
$$

Since $u$ is analytic in $\mathbb{R}^{2}$, applying the reflection principle twice leads to

$$
\begin{aligned}
u\left(\operatorname{Rot}_{\frac{2 \pi}{D \#}}(x)\right)=u\left(\mathrm{R}_{l_{1}} \mathrm{R}_{l_{2}}(x)\right) & =\mp \mathrm{R}_{l_{1}}\left(u\left(\mathrm{R}_{l_{2}}(x)\right)\right) \\
& =\mp \mp \mathrm{R}_{l_{1}} \mathrm{R}_{l_{2}}(u(x))=\operatorname{Rot}_{\frac{2 \pi}{D \#}}(u(x)) .
\end{aligned}
$$

Therefore, the total field $u$ remains rotationally invariant in the sense of (37), which together with Lemma 1 implies the relations $\operatorname{Rot}_{\frac{2 \pi}{D \#}}(\mathcal{P})=\mathcal{P}, \operatorname{Rot}_{\frac{2 \pi}{D \#}}(\mathcal{S})=\mathcal{S}$.

Remark 5. Combining Lemma 6 (i) and Lemma 7, we obtain that

$$
\begin{aligned}
& G_{\boldsymbol{P}}:=\left\{\operatorname{Rot}_{\frac{2 j \pi}{D \#}}(\boldsymbol{P}): j=1,2, \cdots, D^{\#}\right\} \subset \mathcal{P}, \quad \forall \boldsymbol{P} \in \mathcal{P}, \\
& G_{S}:=\left\{\operatorname{Rot}_{\frac{2 j \pi}{D \#}}(\boldsymbol{S}): j=1,2, \cdots, D^{\#}\right\} \subset \mathcal{S}, \quad \forall \boldsymbol{S} \in \mathcal{S} .
\end{aligned}
$$

In addition, if $\operatorname{Rot}_{\frac{2 j \pi}{D \#}}\left(\boldsymbol{P}_{n}\right)=\boldsymbol{P}_{m}$ for some $n, m \in P$, or $\operatorname{Rot}_{\frac{2 j \pi}{D \#}}\left(\boldsymbol{S}_{n}\right)=\boldsymbol{S}_{m}$ for some $n, m \in S, 1 \leq j \leq D^{\#}$, then it holds that

$$
A_{p, m} \boldsymbol{P}_{m}=A_{p, n} \operatorname{Rot}_{\frac{2 j \pi}{D \#}}\left(\boldsymbol{P}_{n}\right), \quad \text { or } \quad A_{s, m} \boldsymbol{S}_{m}^{\perp}=A_{s, n} \operatorname{Rot}_{\frac{2 j \pi}{D \#}}\left(\boldsymbol{S}_{n}^{\perp}\right) .
$$


LEMMA 8. The total field only consists of the compressional part, or equivalently, $\mathcal{S}=\emptyset$.

Proof. For all $\mathbf{S} \in \mathcal{S}$, the set $G_{\mathbf{S}}$ defined in (39) consists of the vertices of some $D^{\#}$-sided regular polygon centered at the origin. If $D^{\#} \geq 3$, then one element of $G_{\mathbf{S}}$ must have a negative $x_{2}$-component, which is impossible due to Remark 4 (ii). Thus $D^{\#}=2$, i.e., $D=\left\{L_{1}, L_{2}\right\}$ with $L_{1} \perp L_{2}$, where $L_{j}(j=1,2)$ are two third (fourth) kind lines extending two line segments of $\Lambda$. Recalling that $\operatorname{Rot}_{\pi}(\mathcal{S})=\mathcal{S}$ if $D^{\#}=2$, and that the second components of the elements in $\mathcal{S}$ are all non-negative, we obtain that $\mathcal{S}=\left\{\left(k_{s}, 0\right),\left(-k_{s}, 0\right)\right\}$. It is seen from $\mathrm{R}_{L_{j}}(\mathcal{S})=\mathcal{S}(j=1,2)(\operatorname{Lemma} 6(i))$ that one of the lines $L_{1}, L_{2}$ must be parallel to the $x_{2}$-axis, contradicting the fact that $\Lambda$ is given by the graph of a function. Thus $\mathcal{S}=\emptyset$.

It follows from the proof of Lemma 8 that we may have $\mathcal{S}=\left\{\left(k_{s}, 0\right),\left(-k_{s}, 0\right)\right\}$ for polygonal grating profiles which are not necessarily given by the graphs of piecewise linear functions. By Lemma 8, we only need to consider the elements in $\mathcal{P}$, which remain invariant under the rotation $\operatorname{Rot}_{\frac{2 \pi}{D \#}}$.

\section{LEMMA 9.}

(i) If $D^{\#}=2$, then

(a) Under the boundary conditions of the third kind,

$$
\begin{aligned}
& \mathcal{P}=\left\{(\alpha,-\beta),(-\alpha, \beta),\left(k_{p}, 0\right),\left(-k_{p}, 0\right)\right\}, \quad \text { if } \pi_{p} \neq \emptyset, \\
& \mathcal{P}=\{(\alpha,-\beta),(-\alpha, \beta)\}, \quad \text { if } \pi_{p}=\emptyset .
\end{aligned}
$$

(b) Under the boundary conditions of the fourth kind,

$$
\pi_{p} \neq \emptyset, \quad \mathcal{P}=\left\{(\alpha,-\beta),(-\alpha, \beta),\left(k_{p}, 0\right),\left(-k_{p}, 0\right)\right\} .
$$

(ii) If $D^{\#} \geq 3$, then $\mathcal{P}=G_{\boldsymbol{P}_{\kappa}}:=\left\{\operatorname{Rot}_{\frac{2 n \pi}{D^{\#}}}\left(\boldsymbol{P}_{\kappa}\right): n=1,2, \cdots, D^{\#}\right\}$.

Here $\pi_{p}:=\left\{n \in \mathbb{Z}: \beta_{n}(\theta)=0\right\}$.

Proof. ( $i$ ) Assume $D^{\#}=2$. The assertion $(i)(a)$ follows directly from Remark 4 (ii) and the fact that $\operatorname{Rot}_{\pi}(\mathbf{P})=-\mathbf{P} \in \mathcal{P}$ for any $\mathbf{P} \in \mathcal{P}$. To prove $(i)(b)$, we only need to exclude the case $\pi_{p}=\emptyset$. By Lemma $5(i i)$, we may assume $D=\left\{L_{1}, L_{2}\right\}$ with $L_{1} \perp L_{2}$. If $\pi_{p}=\emptyset$, then $\mathcal{P}=\{(\alpha,-\beta),(-\alpha, \beta)\}$. Thus, we obtain from $\mathrm{R}_{L_{j}}(\mathcal{P})=\mathcal{P}$ $(j=1,2)$ that one of the lines $L_{1}, L_{2}$ must pass through $\mathbf{P}_{\kappa}$, which contradicts Lemma 6 (iii). This proves the first assertion.

(ii) Note that $G_{\mathbf{P}_{\kappa}}$ consists of the vertices of some $D^{\#}$-sided regular polygon centered at the origin. If there exists some $\mathbf{P} \in \mathcal{P} \backslash G_{\mathbf{P}_{\kappa}}$, then at least one element of $G_{\mathbf{P}}$ has a negative $x_{2}$-component if $D^{\#} \geq 3$. However, this is impossible since $G_{\mathbf{P}_{\kappa}} \cap G_{\mathbf{P}}=\emptyset$ and only $\mathbf{P}_{\kappa} \in G_{\mathbf{P}_{\kappa}} \subset \mathcal{P}$ has a negative $x_{2}$-component, $-\beta$.

We proceed to investigate $D^{\#}$. Denote the straight line which passes through the origin and makes the angle $\varphi$ with the positive $x_{1}$-axis by

$$
L_{\varphi}:=\{(t \cos \varphi, t \sin \varphi): t \in \mathbb{R}, \varphi \in[0,2 \pi)\} .
$$

LEMMA 10. (i) In the case of the boundary conditions of the third kind, $2 \leq$ $D^{\#} \leq 4$.

(ii) In the case of the boundary conditions of the fourth kind, $D^{\#}=2$. 
Proof. (i) If $D^{\#} \geq 5$, then $\mathcal{P}=G_{\mathbf{P}_{\kappa}}$ by Lemma 9 (ii), and thus there are at least two elements of $G_{\mathbf{P}_{\kappa}}$, each of which has a negative $x_{2}$-component. However, this contradicts Remark 4 and proves the lemma in the case of the third kind boundary conditions.

(ii) Under the boundary conditions of the fourth kind, we exclude the cases $D^{\#}=3$ and $D^{\#}=4$, using the fact that $\mathrm{R}_{l}(\mathbf{P}) \neq \mathbf{P}$ for any $\mathbf{P} \in \mathcal{P}, l \in D$ (see Lemma 6 (iii)), i.e., each line of $D$ does not pass through a point of $\mathcal{P}$.

If $D^{\#}=3$, then by Lemma $9, \mathcal{P}=\left\{\mathbf{P}_{\kappa}, \operatorname{Rot}_{\frac{2 \pi}{3}}\left(\mathbf{P}_{\kappa}\right)\right.$, $\left.\operatorname{Rot}_{\frac{4 \pi}{3}}\left(\mathbf{P}_{\kappa}\right)\right\}$. Since $\operatorname{R}_{l}(\mathcal{P})=$ $\mathcal{P}$ for all $l \in D$, we obtain that

$$
D=\left\{L_{\theta-\frac{\pi}{2}}, L_{\theta+\frac{\pi}{6}}, L_{\theta+\frac{5 \pi}{6}}\right\}
$$

noting that the angle formed by the vector $\overrightarrow{O \mathbf{P}_{\kappa}}$ and the positive $x_{1}$-axis is $\theta-\frac{\pi}{2}$. This implies that each line of $D$ goes through a point of $\mathcal{P}$, which is impossible.

If $D^{\#}=4$, then $\mathcal{P}=\left\{\mathbf{P}_{\kappa}, \operatorname{Rot}_{\frac{\pi}{2}}\left(\mathbf{P}_{\kappa}\right), \operatorname{Rot}_{\pi}\left(\mathbf{P}_{\kappa}\right)\right.$, Rot $\left.-\frac{\pi}{2}\left(\mathbf{P}_{\kappa}\right)\right\}$. Since only one element, $\mathbf{P}_{\kappa}=(\alpha,-\beta)=k_{p}(\sin \theta,-\cos \theta)$, has a negative $x_{2}$-component, we have

$$
\theta=0, \quad \mathcal{P}=\left\{\left(0, k_{p}\right),\left(-k_{p}, 0\right),\left(k_{p}, 0\right),\left(0,-k_{p}\right)\right\},
$$

which together with Lemma 5 and Lemma $6(i)$ gives that

$$
D=\left\{L_{0}, L_{\frac{\pi}{4}}, L_{-\frac{\pi}{4}}, L_{\frac{\pi}{2}}\right\}
$$

Hence, the elements belonging to $\mathcal{P}$ are all located on the equi-angular system formed by the lines in $D$. This is again a contradiction. The proof of the lemma is thus complete.

REMARK 6. Under the boundary conditions of the third kind, the elements of $D$ are already given in (40) for $D^{\#}=3$, and in (42) for $D^{\#}=4$, respectively. An argument similar to the proof of Lemma 10 (ii) can be employed to determine the elements of $D$ when $D^{\#}=2$. More precisely, in the case of $D^{\#}=2$, we deduce from Lemma $9(i)$ and $\mathrm{R}_{L}(\mathcal{P})=\mathcal{P}, \forall L \in D$, that

$$
D= \begin{cases}\left\{L_{\theta}, L_{\theta+\frac{\pi}{2}}\right\} & \text { if } \mathcal{P}=\{(\alpha,-\beta),(-\alpha, \beta)\} \\ \left\{L_{\frac{\theta}{2}+\frac{\pi}{4}}, L_{\frac{\theta}{2}-\frac{\pi}{4}}\right\} & \text { if } \mathcal{P}=\left\{(\alpha,-\beta),(-\alpha, \beta),\left(k_{p}, 0\right),\left(-k_{p}, 0\right)\right\}\end{cases}
$$

6.2. Uniqueness under the boundary conditions of the fourth kind. Define a class of unidentifiable polygonal grating profiles $\mathcal{D}_{2}\left(\theta, k_{p}\right)$ by

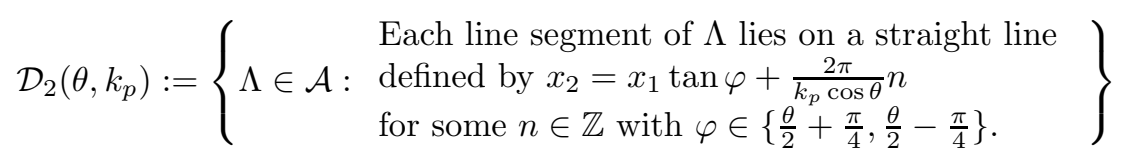

if $k_{p}(1 \pm \sin \theta) \in \mathbb{Z}$, and by $\mathcal{D}_{2}\left(\theta, k_{p}\right):=\emptyset$ otherwise. Suppose $A_{1} A_{2}$ is a line segment of $\Lambda \in \mathcal{D}_{2}\left(\theta, k_{p}\right)$ connecting two corner points $A_{1}$ and $A_{2}$, and $\varphi \in\left[-\frac{\pi}{2}, \frac{\pi}{2}\right)$ is the angle formed by $A_{1} A_{2}$ and the positive $x_{1}$-axis. It follows from the definition of $\mathcal{D}_{2}\left(\theta, k_{p}\right)$ that either $\varphi=\frac{\theta}{2}+\frac{\pi}{4}$ or $\varphi=\frac{\theta}{2}-\frac{\pi}{4}$. If $\varphi=\frac{\theta}{2} \pm \frac{\pi}{4}$, then it can be derived that $\left|A_{1} A_{2}\right|=\frac{\pi}{k \cos \left(\frac{\theta}{2} \pm \frac{\pi}{4}\right)} n^{ \pm}$for some $n^{ \pm} \in \mathbb{N}$. Moreover, $\mathcal{D}_{2}\left(\theta, k_{p}\right) \neq \emptyset$ for all $k_{p}$ and $\theta$ satisfying $k_{p}(1 \pm \sin \theta) \in \mathbb{Z}$ (see Lemma 11), and a Rayleigh frequency of the compressional part always occurs in this case. 
Let us now give the main theorem for the fourth kind boundary conditions.

THEOREM 4. Let $\Lambda_{1}, \Lambda_{2} \in \mathcal{A}$ such that one corner point of $\Lambda_{1}$ or $\Lambda_{2}$ is located at the origin. Assume the boundary conditions of the fourth kind are imposed on $\Lambda_{j}, j=1,2$. If the relation

$$
u_{1}\left(x_{1}, b ; \theta\right)=u_{2}\left(x_{1}, b ; \theta\right), \quad \forall x_{1} \in(0,2 \pi)
$$

holds for one incident pressure wave with incident angle $\theta \in\left(-\frac{\pi}{2}, \frac{\pi}{2}\right)$, then one of the following cases must occur:

(i) $\Lambda_{1}=\Lambda_{2}$.

(ii) $\Lambda_{1}, \Lambda_{2} \in \mathcal{D}_{2}\left(\theta, k_{p}\right), \pi_{p}=\emptyset$, and the total field takes the form

$$
\begin{aligned}
& \qquad \begin{array}{l}
u(x)=\hat{\theta} \exp \left(i k_{p} x \cdot \hat{\theta}\right)-\hat{\theta} \exp \left(-i k_{p} x \cdot \hat{\theta}\right) \\
-e_{1} \exp \left(i k_{p} x_{1}\right)+e_{1} \exp \left(-i k_{p} x_{1}\right) \quad \text { in } \mathbb{R}^{2},
\end{array} \\
& \text { where } u=u_{j}(j=1,2), e_{1}=(1,0)^{\top} .
\end{aligned}
$$

Proof of Theorem 4. Assuming $\Lambda_{1} \neq \Lambda_{2}$ and relying on the reflection principle and the rotational invariance of the propagating directions of the compressional part, we shall prove the second assertion. It follows from Lemma 8 that $\mathcal{S}=\emptyset$, and from Lemmas 9 and 10 and Remark 6 that $\pi_{p} \neq \emptyset, D^{\#}=2$ under the fourth kind of boundary conditions. More specifically, we have

$$
D=\left\{L_{\frac{\theta}{2}+\frac{\pi}{4}}, L_{\frac{\theta}{2}-\frac{\pi}{4}}\right\} \text { and } \mathcal{P}=\left\{(\alpha,-\beta),(-\alpha, \beta),\left(k_{p}, 0\right),\left(-k_{p}, 0\right)\right\}
$$

Furthermore, by Lemmas 5 and 6 , we obtain that each line of $D$ does not pass through a point of $\mathcal{P}, \mathrm{R}_{l}(\mathcal{P})=\mathcal{P}$ for all $l \in D$, and that $L_{\frac{\theta}{2}+\frac{\pi}{4}} \perp L_{\frac{\theta}{2}-\frac{\pi}{4}}$, implying

(47) $\left\{\mathrm{R}_{l}\left(\mathbf{P}_{\kappa}\right): l \in D\right\}=\left\{\mathrm{R}_{l}\left(-\mathbf{P}_{\kappa}\right): l \in D\right\}=\left\{\left(k_{p}, 0\right),\left(-k_{p}, 0\right)\right\}, \operatorname{Rot}_{\pi}\left(\mathbf{P}_{\kappa}\right)=-\mathbf{P}_{\kappa}$.

Note that $\mathbf{P}_{\kappa}=(\alpha,-\beta)=\left(\alpha_{0},-\beta_{0}\right)=k_{p} \hat{\theta}$. In view of Lemma 3 (ii) and Lemma 6 (ii), the identities in (47) give rise to the desired explicit representation (45) for both $u_{1}$ and $u_{2}$. It remains to prove $\Lambda_{1}, \Lambda_{2} \in \mathcal{D}_{2}\left(\theta, k_{p}\right)$.

Denote by $l:=\left\{x \in \mathbb{R}^{2}: x_{2}=k x_{1}+c\right\}$ a straight line extending some line segment of $\Lambda\left(\Lambda=\Lambda_{1}\right.$ or $\left.\Lambda_{2}\right)$. Then the solution $u$ defined in (45) satisfies the boundary conditions of the fourth kind on $l$, and thus by the reflection principle,

$$
u(x)+\mathrm{R}_{l^{\prime}}\left(u\left(\mathrm{R}_{l}(x)\right)\right)=0 \text { in } \mathbb{R}^{2},
$$

where $l^{\prime}:=\left\{x \in \mathbb{R}^{2}: x_{2}=k x_{1}\right\}$. By a coordinate translation, we find that the function $v(x):=u\left(x_{1}, x_{2}+c\right)$ satisfies the Navier equation with the fourth kind boundary conditions on $l^{\prime}$, and thus

$$
v(x)+\mathrm{R}_{l^{\prime}}\left(v\left(\mathrm{R}_{l^{\prime}}(x)\right)\right)=0 \text { in } \mathbb{R}^{2} .
$$

This together with Lemma 14 gives the relation $\mathrm{R}_{l^{\prime}}(\mathcal{P})=\mathcal{P}$. Recalling the elements in $\mathcal{P}$, we obtain that $l^{\prime}$ coincides with one of the lines $L_{\frac{\theta}{2}+\frac{\pi}{4}}, L_{\frac{\theta}{2}-\frac{\pi}{4}}$. On the other hand, since $v$ can be written as

$$
\begin{aligned}
v(x)= & \hat{\theta} \exp \left(i k_{p} x \cdot \hat{\theta}\right) \exp (-i \beta c)-\hat{\theta} \exp \left(-i k_{p} x \cdot \hat{\theta}\right) \exp (i \beta c) \\
& -e_{1} \exp \left(i k_{p} x_{1}\right)+e_{1} \exp \left(-i k_{p} x_{1}\right)
\end{aligned}
$$


combining the first identity in (47) and Lemma 6 (ii) yields that $\exp (i \beta c)=$ $\exp (-i \beta c)=1$, or equivalently,

$$
c=\frac{2 \pi}{\beta} n=\frac{2 \pi}{k_{p} \cos \theta} n, \text { for some } n \in \mathbb{Z} .
$$

To summarize, we have deduced that $l$ can be represented as

$$
l=\left\{x \in \mathbb{R}^{2}: x_{2}=x_{1} \tan \varphi+\frac{2 \pi}{k_{p} \cos \theta} n \text { for some } n \in \mathbb{Z} \text { with } \varphi \in\left\{\frac{\theta}{2}+\frac{\pi}{4}, \frac{\theta}{2}-\frac{\pi}{4}\right\}\right\} .
$$

Finally, it is seen from Lemma 4 and (46) that $k_{p}(1 \pm \sin \theta) \in \mathbb{Z}$, which finishes the proof of $\Lambda_{1}, \Lambda_{2} \in \mathcal{D}_{2}\left(\theta, k_{p}\right)$. The proof of the theorem is complete.

It is seen from the proof of Theorem 4 that each element of $\mathcal{D}_{2}\left(\theta, k_{p}\right)$ generates the same total field of the form (45). In the following, we will show that, for each angle $\theta$ satisfying $k_{p}(1 \pm \sin \theta) \in \mathbb{Z}, \mathcal{D}_{2}\left(\theta, k_{p}\right)$ contains at least two elements, and thus the corresponding counterexample to uniqueness with one incident pressure wave can be constructed .

Lemma 11. For all $k_{p}$ and $\theta$ satisfying $k_{p}(1 \pm \sin \theta) \in \mathbb{Z}, \mathcal{D}_{2}\left(\theta, k_{p}\right)$ is not empty and contains at least two different grating profiles.

Proof. Let $\varphi_{1}=\frac{\theta}{2}+\frac{\pi}{4}, \varphi_{2}=\frac{\theta}{2}-\frac{\pi}{4}$, and let $\Lambda_{i}$ be the $2 \pi$-periodic extensions of $\left.\Lambda_{i}\right|_{(0,2 \pi)}(i=1,2)$ defined by

$$
\begin{aligned}
& \Lambda_{1}: x_{2}=\left\{\begin{array}{ll}
x_{1} \tan \varphi_{1} & x_{1} \in\left(0, T_{1}\right), \\
\left(x_{1}-2 \pi\right) \tan \varphi_{2} & x_{1} \in\left[T_{1}, 2 \pi\right)
\end{array} \text { with } T_{1}=\frac{2 \pi \tan \varphi_{2}}{\tan \varphi_{2}-\tan \varphi_{1}},\right. \\
& \Lambda_{2}: x_{2}=\left\{\begin{array}{ll}
x_{1} \tan \varphi_{2} & x_{1} \in\left(0, T_{2}\right), \\
\left(x_{1}-2 \pi\right) \tan \varphi_{1} & x_{1} \in\left[T_{2}, 2 \pi\right)
\end{array} \text { with } T_{2}=\frac{2 \pi \tan \varphi_{1}}{\tan \varphi_{1}-\tan \varphi_{2}} .\right.
\end{aligned}
$$

It is easy to verify by using $\tan \frac{\varphi}{2}=(1-\cos \varphi) / \sin \varphi$ that

$$
k_{p} \cos \theta \tan \left(\frac{\theta}{2} \pm \frac{\pi}{4}\right)=k_{p} \cos \theta \frac{1-\cos \left(\theta \pm \frac{\pi}{2}\right)}{\sin \left(\theta \pm \frac{\pi}{2}\right)}=k_{p}(1 \pm \sin \theta) \in \mathbb{Z}
$$

which implies that both $\Lambda_{1}$ and $\Lambda_{2}$ belong to $\mathcal{D}_{2}\left(\theta, k_{p}\right)$. Thus there exist at least two grating profiles in $\mathcal{D}_{2}\left(\theta, k_{p}\right)$ if both $k_{p}(1+\sin \theta)$ and $k_{p}(1-\sin \theta)$ are integers.

Essentially, given $k_{p}$ and $\theta$ satisfying $k_{p}(1 \pm \sin \theta) \in \mathbb{Z}$, if $\Lambda$ is $2 \pi$-periodic with respect to $x_{1}$-direction and lies on the rectangular grids generated by the $2 \pi$-periodic extensions of $x_{2}=x_{1} \tan \left(\frac{\theta}{2} \pm \frac{\pi}{4}\right)$, then $\Lambda \in \mathcal{D}_{2}\left(\theta, k_{p}\right)$ (see Figure 1 ). The elements in $\mathcal{D}_{2}\left(\theta, k_{p}\right)$ provide non-uniqueness examples for the inverse problem with the fourth kind boundary conditions.

Counterexample 1 . Under the boundary conditions of the fourth kind, one incident pressure wave is not enough to uniquely determine $\Lambda \in \mathcal{A}$.

Let $\Lambda_{1}$ and $\Lambda_{2}$ be the grating profiles defined in the proof of Lemma 11 with $\theta=-\pi / 6$ and $k_{p}=2$; see Figure 1 . Then the total fields

$$
u_{1}(x)=u_{2}(x)=\left(\begin{array}{c}
-1 / 2 \\
-\sqrt{3} / 2
\end{array}\right) e^{i\left(-x_{1}-\sqrt{3} x_{2}\right)}+\left(\begin{array}{c}
1 / 2 \\
\sqrt{3} / 2
\end{array}\right) e^{i\left(x_{1}+\sqrt{3} x_{2}\right)}-\left(\begin{array}{c}
-1 \\
0
\end{array}\right) e^{-2 i x_{1}}-\left(\begin{array}{l}
1 \\
0
\end{array}\right) e^{2 i x_{1}}
$$




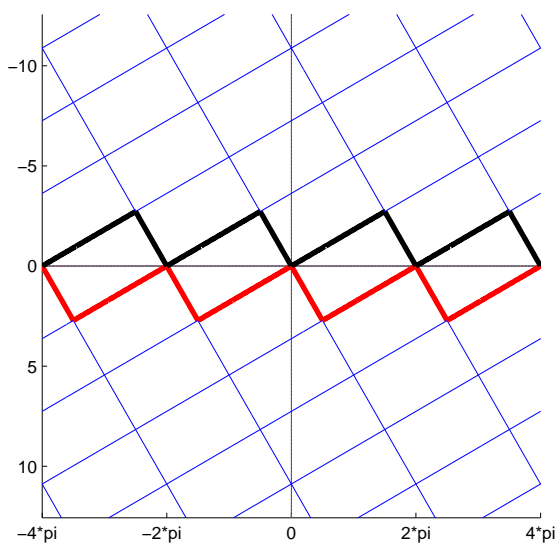

FIG. 1. $\mathcal{D}_{2}\left(\theta, k_{p}\right)$ with $\theta=-\frac{\pi}{6}, k_{p}=2$.

satisfy the fourth kind boundary conditions on both $\Lambda_{1}$ and $\Lambda_{2}$, the $\alpha$-quasi-periodicity condition with $\alpha=k_{p} \sin \theta=-1$ and the Rayleigh expansion (10). Thus the nearfield data $u\left(x_{1}, b\right)$ from one incident plane pressure wave is not sufficient to determine $\Lambda \in \mathcal{A}$ uniquely.

REMARK 7. Let $\Lambda \in \mathcal{A}$ have one corner point at the origin. The following results can be obtained directly from Theorem 4 .

(i) Given the a priori information that $\Lambda$ does not belong to $\mathcal{D}_{2}\left(\theta, k_{p}\right)$, the data of the total field on $\Gamma_{b}$ from one incident pressure wave (with the incident angle $\theta)$ are always enough to uniquely determine $\Lambda$.

(ii) Consider a fixed incident angle $\theta \in\left(-\frac{\pi}{2}, \frac{\pi}{2}\right)$. If $\mathcal{D}_{2}\left(\theta, k_{p}\right)=\emptyset$, then one incident pressure wave with the incident angle $\theta$ uniquely determines $\Lambda \in \mathcal{A}$. Note that $\mathcal{D}_{2}\left(\theta, k_{p}\right)=\emptyset$ if one of the numbers $k_{p}(1+\sin \theta), k_{p}(1-\sin \theta)$ is not an integer. In particular, if $\pi_{p}=\emptyset$, then both $k_{p}(1+\sin \theta)$ and $k_{p}(1-\sin \theta)$ are not integers. Thus, one incident pressure wave is always enough if Rayleigh frequencies of the compressional part are excluded.

(iii) Two incident pressure waves are sufficient to uniquely determine $\Lambda \in \mathcal{A}$ since

$$
\mathcal{D}_{2}\left(\theta_{1}, k_{p}\right) \cap \mathcal{D}_{2}\left(\theta_{2}, k_{p}\right)=\emptyset, \quad \theta_{1} \neq \theta_{2} .
$$

6.3. Uniqueness under the boundary conditions of the third kind. Before stating the main theorem for the third kind boundary conditions, we define the following three classes of polygonal periodic structures. If $2 k_{p} \sin \theta \in \mathbb{Z}, \mathcal{N}_{2}\left(\theta, k_{p}\right)$ is defined by

$$
\mathcal{N}_{2}\left(\theta, k_{p}\right):=\left\{\begin{array}{ll} 
& \text { Each line segment of } \Lambda \text { lies on a straight line defined } \\
\Lambda \in \mathcal{A}: & \text { by } x_{2}=x_{1} \tan \theta+\frac{\pi}{k_{p} \cos \theta} n \text { for some } n \in \mathbb{Z}, \text { or } \\
& x_{2}=x_{1} \tan \left(\theta+\frac{\pi}{2}\right)+c \text { for some } c \in \mathbb{R} .
\end{array}\right\},
$$

and if $2 k_{p} \sin \theta \notin \mathbb{Z}, \mathcal{N}_{2}\left(\theta, k_{p}\right):=\emptyset$. The set $\mathcal{N}_{3}\left(\theta, k_{p}\right)$ is defined by

$\mathcal{N}_{3}\left(\theta, k_{p}\right):=\left\{\begin{array}{ll} & \text { Each line segment of } \Lambda \text { lies on a straight line defined by } \\ \Lambda \in \mathcal{A}: & x_{2}=x_{1} \tan \varphi+\frac{2 \pi}{k_{p} \sqrt{3} \cos \varphi} n \text { for some } n \in \mathbb{Z}, \text { with } \\ \varphi \in\left\{\theta+\frac{5 \pi}{6}, \theta+\frac{\pi}{6}, \theta-\frac{\pi}{2}\right\} .\end{array}\right\}$, 
if $\theta \in\left[-\frac{\pi}{6}, \frac{\pi}{6}\right]$ and $k_{p} \sqrt{3} \sin \left(\frac{\pi}{6} \pm \theta\right) \in \mathbb{Z}$, and by $\mathcal{N}_{3}\left(\theta, k_{p}\right):=\emptyset$ otherwise. Finally,

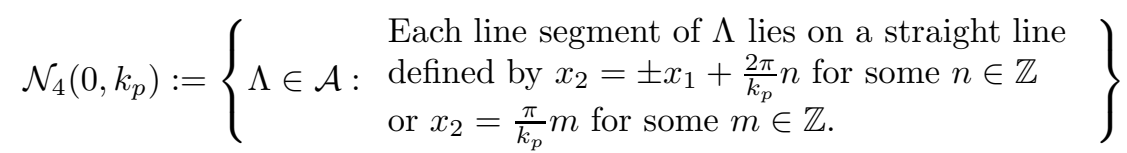

if $\theta=0$ and $k_{p} \in \mathbb{Z}$, and $\mathcal{N}_{4}\left(0, k_{p}\right):=\emptyset$ otherwise.

ThEOREM 5. Let $\Lambda_{1}, \Lambda_{2} \in \mathcal{A}$ be such that one of the profiles $\Lambda_{1}, \Lambda_{2}$ has a corner point at the origin. Assume the third kind boundary conditions are imposed on $\Lambda_{j}, j=$ 1,2 . If the relation (44) holds for one incident pressure wave with incident angle $\theta \in\left(-\frac{\pi}{2}, \frac{\pi}{2}\right)$, then one of the following cases must occur:

(i) $\Lambda_{1}=\Lambda_{2}$.

(ii) (a) $\Lambda_{1}, \Lambda_{2} \in \mathcal{N}_{2}\left(\theta, k_{p}\right), \pi_{p}=\emptyset$, and the total field takes the form

$$
u(x)=\hat{\theta} \exp \left(i k_{p} x \cdot \hat{\theta}\right)-\hat{\theta} \exp \left(-i k_{p} x \cdot \hat{\theta}\right) \quad \text { in } \quad \mathbb{R}^{2} .
$$

(b) $\Lambda_{1}, \Lambda_{2} \in \mathcal{D}_{2}\left(\theta, k_{p}\right), \pi_{p} \neq \emptyset$, and the total field takes the form

$$
\begin{aligned}
u(x)= & \hat{\theta} \exp \left(i k_{p} x \cdot \hat{\theta}\right)-\hat{\theta} \exp \left(-i k_{p} x \cdot \hat{\theta}\right) \\
& +e_{1} \exp \left(i k_{p} x_{1}\right)-e_{1} \exp \left(-i k_{p} x_{1}\right) \text { in } \mathbb{R}^{2} .
\end{aligned}
$$

(iii) $\Lambda_{1}, \Lambda_{2} \in \mathcal{N}_{3}\left(\theta, k_{p}\right)$ with $\theta \in\left[-\frac{\pi}{6}, \frac{\pi}{6}\right]$. In this case, $\pi_{p} \neq \emptyset$ if $\theta=\frac{\pi}{6}$ or $\theta=-\frac{\pi}{6}$, and the total field takes the following form in $\mathbb{R}^{2}$ :

$$
\begin{aligned}
u(x)= & \hat{\theta} \exp \left(i k_{p} x \cdot \hat{\theta}\right)+\operatorname{Rot}_{\frac{2 \pi}{3}}(\hat{\theta}) \exp \left(i k_{p} x \cdot \operatorname{Rot}_{\frac{2 \pi}{3}}(\hat{\theta})\right) \\
& +\operatorname{Rot}_{\frac{4 \pi}{3}}(\hat{\theta}) \exp \left(i k_{p} x \cdot \operatorname{Rot}_{\frac{4 \pi}{3}}(\hat{\theta})\right) .
\end{aligned}
$$

(iv) $\Lambda_{1}, \Lambda_{2} \in \mathcal{N}_{4}\left(0, k_{p}\right), \theta=0, \pi_{p} \neq \emptyset$, and the total field takes the form

$$
\begin{aligned}
u(x)= & -e_{2} \exp \left(-i k_{p} x_{2}\right)+e_{2} \exp \left(i k_{p} x_{2}\right) \\
& +e_{1} \exp \left(i k_{p} x_{1}\right)-e_{1} \exp \left(-i k_{p} x_{1}\right) \text { in } \mathbb{R}^{2} .
\end{aligned}
$$

Here $u=u_{j}(j=1,2), e_{1}=(1,0)^{\top}$ and $e_{2}=(0,1)^{\top}$.

Proof. Assuming $\Lambda_{1} \neq \Lambda_{2}$, we shall prove that one of the cases (ii), (iii) and (iv) must happen. It is seen from Lemma 8 and Lemma 10 that $\mathcal{S}=\emptyset$ and $2 \leq D^{\#} \leq 4$. Moreover, combining Lemmas 9 and 10 and Remark 6 , we obtain the following results.

1. If $D^{\#}=2$ and $\pi_{p}=\emptyset$, then

$$
D=\left\{L_{\theta}, L_{\theta+\frac{\pi}{2}}\right\}, \quad \mathcal{P}=\{(\alpha,-\beta),(-\alpha, \beta)\}=\left\{\mathbf{P}_{\kappa},-\mathbf{P}_{\kappa}\right\}
$$

with the relations

$$
\mathrm{R}_{L_{\theta}}\left(\mathbf{P}_{\kappa}\right)=\mathbf{P}_{\kappa}, \mathrm{R}_{L_{\theta+\frac{\pi}{2}}}\left(\mathbf{P}_{\kappa}\right)=-\mathbf{P}_{\kappa}
$$

From Lemma $6(\mathrm{ii})$ and Lemma 4 , it follows that the total field takes the form $(48)$ and $2 k_{p} \sin \theta \in \mathbb{Z}$.

2. If $D^{\#}=2$ and $\pi_{p} \neq \emptyset$, then an argument similar to the case of the fourth kind boundary conditions yields the same relations as in (46) and (47), which together with Lemma 6 (ii) and Lemma 4 give the explicit expression of $u$ in (49) as well as the relation $k_{p}(1 \pm \sin \theta) \in \mathbb{Z}$. 
3. If $D^{\#}=3$, it follows from (40) and Lemma 9 (ii) that

$$
D=\left\{L_{\theta-\frac{\pi}{2}}, L_{\theta+\frac{\pi}{6}}, L_{\theta+\frac{5 \pi}{6}}\right\}, \mathcal{P}=\left\{\mathbf{P}_{\kappa}, \operatorname{Rot}_{\frac{2 \pi}{3}}\left(\mathbf{P}_{\kappa}\right), \operatorname{Rot}_{\frac{4 \pi}{3}}\left(\mathbf{P}_{\kappa}\right)\right\}
$$

with the relations

$$
\begin{aligned}
\mathrm{R}_{L_{\theta+\frac{5 \pi}{6}}}\left(\mathbf{P}_{\kappa}\right) & =\operatorname{Rot}_{\frac{2 \pi}{3}}\left(\mathbf{P}_{\kappa}\right), \\
\mathrm{R}_{L_{\theta+\frac{\pi}{6}}} & \left(\mathbf{P}_{\kappa}\right)=\operatorname{Rot}_{\frac{4 \pi}{3}}\left(\mathbf{P}_{\kappa}\right), \mathrm{R}_{L_{\theta-\frac{\pi}{2}}}\left(\operatorname{Rot}_{\frac{4 \pi}{3}}\left(\mathbf{P}_{\kappa}\right)\right)=\operatorname{Rot}_{\frac{2 \pi}{3}}\left(\mathbf{P}_{\kappa}\right) .
\end{aligned}
$$

By Lemma 6 (ii), we know that the identity (50) holds in $\mathbb{R}^{2}$.

4. If $D^{\#}=4$, by (42) we have that $\theta=0$, and

$$
D=\left\{L_{0}, L_{\frac{\pi}{4}}, L_{-\frac{\pi}{4}}, L_{\frac{\pi}{2}}\right\}, \mathcal{P}=\left\{\left(0, k_{p}\right),\left(-k_{p}, 0\right),\left(k_{p}, 0\right),\left(0,-k_{p}\right)\right\},
$$

which implies that

$$
\begin{aligned}
& \mathrm{R}_{L_{-\frac{\pi}{4}}}\left\{\left(0,-k_{p}\right)\right\}=\left\{\left(k_{p}, 0\right)\right\}, \quad \mathrm{R}_{L_{\frac{\pi}{4}}}\left\{\left(0,-k_{p}\right)\right\}=\left\{\left(-k_{p}, 0\right)\right\} \\
& \mathrm{R}_{L_{0}}\left\{\left(0,-k_{p}\right)\right\}=\left\{\left(0, k_{p}\right)\right\}
\end{aligned}
$$

Applying Lemma 6 (ii) again gives (51).

The assertions $(i i)(a),(i i)(b),(i i i)$ and $(i v)$ of Theorem 5 are direct consequences of the above assertions $1-4$. We next prove assertion $(i i i)$ in the case of $D^{\#}=3$. To do this, we need to prove that $\Lambda_{1}, \Lambda_{2} \in \mathcal{N}_{3}\left(\theta, k_{p}\right)$.

Firstly, we note that the elements of $\mathcal{P}$ can be written as

$$
\begin{aligned}
\mathbf{P}_{\kappa} & =k_{p}\left(\cos \left(\theta-\frac{\pi}{2}\right), \sin \left(\theta-\frac{\pi}{2}\right)\right), \\
\operatorname{Rot}_{\frac{2 \pi}{3}}\left(\mathbf{P}_{\kappa}\right) & =k_{p}\left(\cos \left(\theta+\frac{\pi}{6}\right), \sin \left(\theta+\frac{\pi}{6}\right)\right), \\
\operatorname{Rot}_{\frac{4 \pi}{3}}\left(\mathbf{P}_{\kappa}\right) & =k_{p}\left(\cos \left(\theta+\frac{5 \pi}{6}\right), \sin \left(\theta+\frac{5 \pi}{6}\right)\right) .
\end{aligned}
$$

Since the second Cartesian components of $\operatorname{Rot}_{\frac{2 \pi}{3}}\left(\mathbf{P}_{\kappa}\right), \operatorname{Rot}_{\frac{4 \pi}{3}}\left(\mathbf{P}_{\kappa}\right)$ are all non-negative, we derive that $\theta+\frac{\pi}{6} \geq 0$ and $\theta+\frac{5 \pi}{6} \leq \pi$, or equivalently, $-\frac{\pi}{6} \leq \theta \leq \frac{\pi}{6}$. Obviously, $\pi_{p} \neq \emptyset$ if $\theta=\frac{\pi}{6}$ or $\theta=-\frac{\pi}{6}$.

Secondly, by the $k_{p} \sin \theta$-quasi-periodicity condition imposed on $u$, we need to check that

$$
\begin{gathered}
k_{p} \cos \left(\theta+\frac{\pi}{6}\right)=k_{p} \sin \theta+n, \text { for some } n \in \mathbb{Z}, \\
k_{p} \cos \left(\theta+\frac{5 \pi}{6}\right)=k_{p} \sin \theta+m, \text { for some } m \in \mathbb{Z},
\end{gathered}
$$

from which $k_{p} \sqrt{3} \sin \left(\frac{\pi}{6} \pm \theta\right) \in \mathbb{Z}$ follows.

Denote by $l:=\left\{x \in \mathbb{R}^{2}: x_{2}=x_{1} \tan \varphi+c\right\}$ a straight line extending some line segment of $\Lambda$, on which the solution $u$ defined in (50) satisfies the boundary conditions of the third kind. Finally, it remains to prove that $\varphi \in\left\{\theta+\frac{\pi}{2}, \theta+\frac{\pi}{6}, \theta+\frac{5 \pi}{6}\right\}$ and $c=\frac{2 \pi}{k_{p} \sqrt{3} \cos \varphi} n$ for some $n \in \mathbb{Z}$.

Analogously to the proof of Theorem 4, we claim that the function $v(x):=$ $u\left(x_{1}, x_{2}+c\right)$ satisfies the Navier equation with the boundary conditions of the third kind on $l^{\prime}$. By the reflection principle, we have

$$
v(x)-\mathrm{R}_{l^{\prime}}\left(v\left(\mathrm{R}_{l^{\prime}}(x)\right)\right)=0 \text { in } \mathbb{R}^{2},
$$


where $l^{\prime}:=\left\{x \in \mathbb{R}^{2}: x_{2}=x_{1} \tan \varphi\right\}$. This together with Lemma 1 leads to the relation $\mathrm{R}_{l^{\prime}}(\mathcal{P})=\mathcal{P}$, and thus $\varphi \in\left\{\theta+\frac{\pi}{2}, \theta+\frac{\pi}{6}, \theta+\frac{5 \pi}{6}\right\}$. By (53)-(55), $v$ can be written as

$$
\begin{aligned}
v(x)= & \hat{\theta} \exp \left(i k_{p} x \cdot \hat{\theta}\right) \exp \left(i c k_{p} \sin \left(\theta-\frac{\pi}{2}\right)\right) \\
& +\operatorname{Rot}_{\frac{2 \pi}{3}}(\hat{\theta}) \exp \left(i k_{p} x \cdot \operatorname{Rot}_{\frac{2 \pi}{3}}(\hat{\theta})\right) \exp \left(i c k_{p} \sin \left(\theta+\frac{\pi}{6}\right)\right) \\
& +\operatorname{Rot}_{\frac{4 \pi}{3}}(\hat{\theta}) \exp \left(i k_{p} x \cdot \operatorname{Rot}_{\frac{4 \pi}{3}}(\hat{\theta})\right) \exp \left(i c k_{p} \sin \left(\theta+\frac{5 \pi}{6}\right)\right) .
\end{aligned}
$$

Without loss of generality, we can assume that $\varphi=\theta+\frac{5 \pi}{6}$, or equivalently, $l^{\prime}=L_{\theta+\frac{5 \pi}{6}}$. Then, combining (52), (56) and Lemma 6 (ii) yields that

$$
\exp \left(i c k_{p} \sin \left(\theta-\frac{\pi}{2}\right)\right)=\exp \left(i c k_{p} \sin \left(\theta+\frac{\pi}{6}\right)\right)
$$

which implies that $c=\frac{2 \pi}{k_{p} \sqrt{3} \cos \left(\theta+\frac{5 \pi}{6}\right)} n$ for some $n \in Z$. Analogously, we can deduce from the previous argument that

$$
\begin{gathered}
c=\frac{2 \pi}{k_{p} \sqrt{3} \cos \left(\theta+\frac{\pi}{6}\right)} n \text { for some } n \in Z, \quad \text { if } l^{\prime}=L_{\theta+\frac{\pi}{6}}, \\
c=\frac{2 \pi}{k_{p} \sqrt{3} \cos \left(\theta-\frac{\pi}{2}\right)} m \quad \text { for some } m \in Z, \quad \text { if } l^{\prime}=L_{\theta-\frac{\pi}{2}} .
\end{gathered}
$$

This proves assertion $($ iii $)$ when $D^{\#}=3$. The other cases when $D^{\#}=2$ or $D^{\#}=4$ can be treated similarly. Special attention should be paid to the case $D^{\#}=4$, for which we obtain that each line segment of $\Lambda$ lies on one of the following straight lines,

$$
x_{1}=\frac{\pi}{k_{p}} n_{1}, x_{2}=\frac{\pi}{k_{p}} n_{2}, x_{2}=x_{1}+\frac{2 \pi}{k_{p}} n_{3}, x_{2}=x_{1}+\frac{2 \pi}{k_{p}} n_{4}
$$

for $n_{j} \in \mathbb{Z}$ with $j=1,2,3,4$. Since all the profiles in $\mathcal{A}$ can be represented by the graph of a piecewise linear function, $\Lambda$ does not contain those line segments which are parallel to the $x_{2}$-axis. This leads to the class $N_{4}\left(0, k_{p}\right)$ defined at the beginning of Section 6.3 for $D^{\#}=4$.

We next give several non-uniqueness examples of (IP) by describing the elements in $\mathcal{N}_{2}\left(\theta, k_{p}\right), \mathcal{N}_{3}\left(\theta, k_{p}\right)$ and $\mathcal{N}_{4}\left(0, k_{p}\right)$, and answer the question in Section 2 of how many incident elastic waves are sufficient to uniquely determine a grating profile from $\mathcal{A}$.

Counterexample 2. If a Rayleigh frequency of the compressional part occurs, two incident pressure waves are not sufficient to uniquely determine a grating profile $\Lambda \in \mathcal{A}$.

Set $k_{p}=2, \theta_{1}=\frac{\pi}{6}$ and $\theta_{2}=-\frac{\pi}{6}$. Let $\left.\Lambda_{1}\right|_{(0,2 \pi)}$ and $\left.\Lambda_{2}\right|_{(0,2 \pi)}$ be defined by the following piecewise linear functions

$$
\begin{aligned}
& \left.\Lambda_{1}\right|_{(0,2 \pi)}: x_{2}= \begin{cases}\sqrt{3} x_{1} & x_{1} \in\left(0, \frac{2}{3} \pi\right), \\
2 \sqrt{3} \pi / 3 & x_{1} \in\left[\frac{2}{3} \pi, \frac{4}{3} \pi\right], \\
-\sqrt{3} x_{1}+2 \sqrt{3} \pi & x_{1} \in\left(\frac{4}{3} \pi, 2 \pi\right),\end{cases} \\
& \left.\Lambda_{2}\right|_{(0,2 \pi)}: x_{2}= \begin{cases}-\sqrt{3} x_{1} & x_{1} \in\left(0, \frac{2}{3} \pi\right), \\
-2 \sqrt{3} \pi / 3 & x_{1} \in\left[\frac{2}{3} \pi, \frac{4}{3} \pi\right], \\
\sqrt{3} x_{1}-2 \sqrt{3} \pi & x_{1} \in\left(\frac{4}{3} \pi, 2 \pi\right),\end{cases}
\end{aligned}
$$


and let $\Lambda_{i}$ be the $2 \pi$-periodic extensions of $\left.\Lambda_{i}\right|_{(0,2 \pi)}(i=1,2)$. One can check that $\Lambda_{1}, \Lambda_{2}$ lie on the grid generated by the lines $x_{2}= \pm \sqrt{3} x_{1}+\frac{2 \pi}{\sqrt{3}} n, x_{2}=\frac{\pi}{\sqrt{3}} m$ with $n, m \in \mathbb{Z}$ (see Figure 2, left), and thus $\Lambda_{1}, \Lambda_{2} \in \mathcal{N}_{3}\left(\frac{\pi}{6}, 2\right) \cap \mathcal{N}_{3}\left(-\frac{\pi}{6}, 2\right)$. Furthermore, the finite Rayleigh expansions

$$
\begin{aligned}
& u_{1}^{(1)}=u_{2}^{(1)}=\left(\begin{array}{c}
1 / 2 \\
-\sqrt{3} / 2
\end{array}\right) e^{i\left(x_{1}-\sqrt{3} x_{2}\right)}+\left(\begin{array}{c}
1 / 2 \\
\sqrt{3} / 2
\end{array}\right) e^{i\left(x_{1}+\sqrt{3} x_{2}\right)}+\left(\begin{array}{c}
-1 \\
0
\end{array}\right) e^{-2 i x_{1}}, \\
& u_{1}^{(2)}=u_{2}^{(2)}=\left(\begin{array}{c}
-1 / 2 \\
-\sqrt{3} / 2
\end{array}\right) e^{-i\left(x_{1}+\sqrt{3} x_{2}\right)}+\left(\begin{array}{c}
-1 / 2 \\
\sqrt{3} / 2
\end{array}\right) e^{-i\left(x_{1}-\sqrt{3} x_{2}\right)}+\left(\begin{array}{c}
1 \\
0
\end{array}\right) e^{2 i x_{1}}
\end{aligned}
$$

all satisfy the Navier equation and the boundary conditions of the third kind on both $\Lambda_{1}$ and $\Lambda_{2}$.
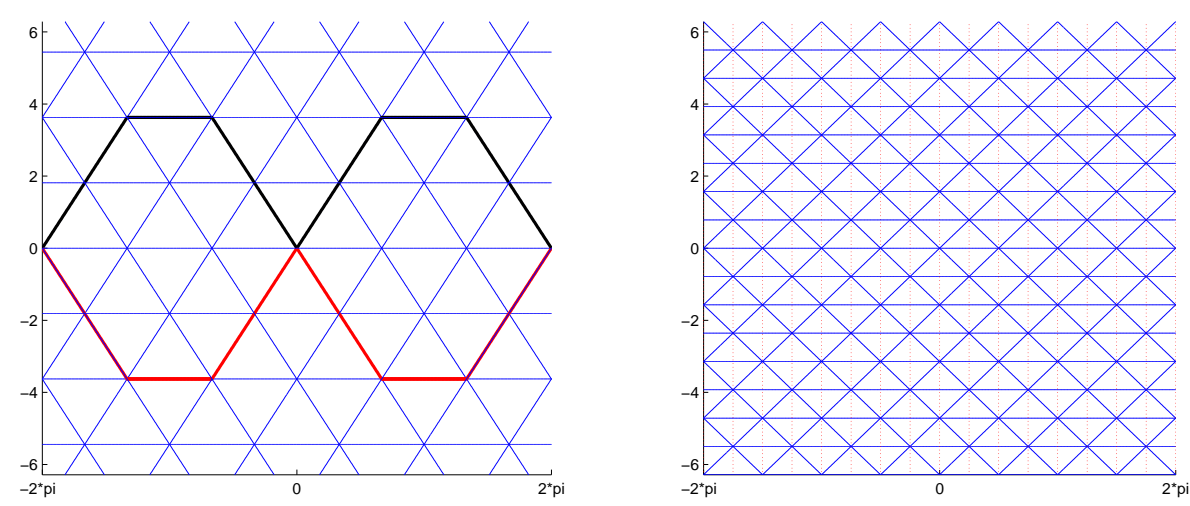

FIG. 2. Left: $\mathcal{N}_{3}\left(\theta, k_{p}\right)$ with $\theta=\frac{\pi}{6}$ or $-\frac{\pi}{6}, k_{p}=2$. Right: the grid on which the profiles of $N_{4}\left(0, k_{p}\right)$ with $k_{p}=4$ are located.

REMARK 8. Analogously to Counterexample 2, a non-uniqueness example for illustrating that one incident pressure wave is not enough to uniquely identify $\Lambda \in \mathcal{A}$ can be constructed from the elements in $\mathcal{N}_{4}\left(0, k_{p}\right)$. In fact, if $\Lambda_{1}, \Lambda_{2} \in \mathcal{A}$ lie on the solid grid indicated in Figure 2 (right), then $\Lambda_{1}, \Lambda_{2} \in \mathcal{N}_{4}\left(0, k_{p}\right)$ with $k_{p}=4$, and thus the total fields for $\Lambda_{1}$ and $\Lambda_{2}$ both take the form (51) with $k_{p}=4$ and $\theta=0$.

Counterexample 3. A grating profile $\Lambda \in \mathcal{A}$ cannot be uniquely determined by two incident pressure waves, in general, even if Rayleigh frequencies of the compressional part for each incident angle are excluded.

It follows from the proof of Theorem 5 (ii) (a) that if $\Lambda_{1}, \Lambda_{2} \in \mathcal{N}_{2}\left(\theta, k_{p}\right) \cap \mathcal{N}_{2}(\theta+$ $\left.\frac{\pi}{2}, k_{p}\right)$ for some $\theta \in\left(-\frac{\pi}{2}, 0\right)$ satisfying $2 k_{p} \sin \theta \in \mathbb{Z}$ and $2 k_{p} \cos \theta \in \mathbb{Z}$, then, for $\theta_{1}=\theta$, $u^{i n}=\hat{\theta}_{1} \exp \left(i k_{p} x \cdot \hat{\theta}_{1}\right)=\hat{\theta} \exp \left(i k_{p} x \cdot \hat{\theta}\right)$, the total fields $u_{1}^{(1)}$ and $u_{2}^{(1)}$ corresponding to $\Lambda_{1}$ and $\Lambda_{2}$ take the form

$$
u_{1}^{(1)}=u_{2}^{(1)}=\hat{\theta} \exp \left(i k_{p} x \cdot \hat{\theta}\right)-\hat{\theta} \exp \left(-i k_{p} x \cdot \hat{\theta}\right) \text { in } \quad \mathbb{R}^{2}
$$

and for $\theta_{2}=\theta+\frac{\pi}{2}, u^{i n}=\hat{\theta}_{2} \exp \left(i k_{p} x \cdot \hat{\theta}_{2}\right)=\hat{\theta}^{\perp} \exp \left(i k_{p} x \cdot \hat{\theta}^{\perp}\right)$, the total fields $u_{1}^{(2)}$ and $u_{2}^{(2)}$ corresponding to $\Lambda_{1}$ and $\Lambda_{2}$ take the form

$$
u_{1}^{(2)}=u_{2}^{(2)}=\hat{\theta}^{\perp} \exp \left(i k_{p} x \cdot \hat{\theta}^{\perp}\right)-\hat{\theta}^{\perp} \exp \left(-i k_{p} x \cdot \hat{\theta}^{\perp}\right) \text { in } \mathbb{R}^{2} .
$$


This implies that two incident pressure waves are not enough to uniquely determine $\Lambda \in \mathcal{A}$ under the third kind boundary conditions. Take $k_{p}=\frac{5}{2}$, choose $\theta$ satisfying $\sin \theta=-\frac{3}{5}, \cos \theta=\frac{4}{5}$, and let the grating profiles $\Lambda_{1}$ and $\Lambda_{2}$ lie on the grid generated by the $2 \pi$-periodic extensions of the lines $x_{2}=-\frac{3}{4} x_{1}, x_{2}=\frac{4}{3} x_{1}$. Then, one may check that $\Lambda_{1}, \Lambda_{2} \in \mathcal{N}_{2}\left(\theta, k_{p}\right) \cap \mathcal{N}_{2}\left(\theta+\frac{\pi}{2}, k_{p}\right)$ with the previously specified $\theta$ and $k_{p}$, and thus obtains a non-uniqueness example for (IP).

Counterexample 4. If a Rayleigh frequency of the compressional part occurs, three incident pressure waves are not sufficient to uniquely determine a grating profile $\Lambda \in \mathcal{A}$.

It can be derived from the definitions of $\mathcal{D}_{2}\left(\theta, k_{p}\right), \mathcal{N}_{2}\left(\theta, k_{p}\right)$ that

$$
\mathcal{N}_{2}\left(\theta_{1}, k_{p}\right) \cap \mathcal{N}_{2}\left(\theta_{2}, k_{p}\right) \cap \mathcal{D}_{2}\left(\theta_{3}, k_{p}\right) \neq \emptyset
$$

if and only if $\theta_{1} \in\left(0, \frac{\pi}{2}\right), \theta_{2} \in\left(-\frac{\pi}{2}, 0\right), \theta_{3} \in\left(-\frac{\pi}{2}, \frac{\pi}{2}\right)$ satisfy

$$
\theta_{2}=\theta_{1}-\frac{\pi}{2}, \theta_{3}=2 \theta_{1}-\frac{\pi}{2}, 2 k_{p} \sin \theta_{1} \in \mathbb{Z}, 2 k_{p} \sin \theta_{2} \in \mathbb{Z}, \quad k_{p}\left(1 \pm \sin \theta_{3}\right) \in \mathbb{Z} .
$$

Thus, if $\Lambda_{1}, \Lambda_{2} \in \mathcal{N}_{2}\left(\theta_{1}, k_{p}\right) \cap \mathcal{N}_{2}\left(\theta_{1}-\frac{\pi}{2}, k_{p}\right) \cap \mathcal{D}_{2}\left(2 \theta_{1}-\frac{\pi}{2}, k_{p}\right)$ for some incident angle $\theta_{1} \in\left(0, \frac{\pi}{2}\right)$ satisfying

$$
2 k_{p} \sin \theta_{1} \in \mathbb{Z}, \quad 2 k_{p} \cos \theta_{1} \in \mathbb{Z}, \quad 4 k_{p}\left(\cos ^{2} \theta_{1}-1\right) \in \mathbb{Z}, \text { with } 2 k_{p} \in \mathbb{Z},
$$

then, for the incident pressure wave $u^{i n}=\exp \left(i k_{p} x \cdot \hat{\theta}_{j}\right)(j=1,2)$, the total fields $u_{1}^{(j)}$ and $u_{2}^{(j)}(j=1,2)$ corresponding to $\Lambda_{1}$ and $\Lambda_{2}$ take the form (48) with $\theta=\theta_{j}$ $(j=1,2)$; and for $u^{i n}=\hat{\theta}_{3} \exp \left(i k_{p} x \cdot \hat{\theta}_{3}\right)$, the total fields $u_{1}^{(3)}$ and $u_{2}^{(3)}$ can be expressed by (49) with $\theta=\theta_{3}$. Note that $\pi_{p} \neq \emptyset$ for the incident angle $\theta_{3}=2 \theta_{1}-\frac{\pi}{2}$.

Let the grating profiles $\left.\Lambda_{i}\right|_{(0,2 \pi)}(i=1,2)$ be defined by the following functions:

$$
\begin{aligned}
& \Lambda_{1}: x_{2}=\left\{\begin{array}{ll}
-\frac{3}{4}\left(x_{1}-2 \pi\right) & x_{1} \in\left[T_{1}, 2 \pi\right), \\
\frac{4}{3} x_{1} & x_{1} \in\left[0, T_{1}\right)
\end{array} \text { with } T_{1}=\frac{18}{25} \pi,\right. \\
& \Lambda_{2}: x_{2}=\left\{\begin{array}{ll}
\frac{4}{3}\left(x_{1}-2 \pi\right) & x_{1} \in\left[T_{2}, 2 \pi\right), \\
-\frac{3}{4} x_{1} & x_{1} \in\left[0, T_{2}\right)
\end{array} \text { with } T_{2}=\frac{32}{25} \pi,\right.
\end{aligned}
$$

and let $\Lambda_{i}$ be the $2 \pi$-periodic extensions of $\left.\Lambda_{i}\right|_{(0,2 \pi)}(i=1,2)$. Take $k_{p}=\frac{25}{2}$, choose $\theta_{j}$ satisfying

$$
\sin \theta_{1}=\frac{4}{5}, \quad \sin \theta_{2}=-\frac{3}{5} \text { and } \sin \theta_{3}=\frac{7}{25} .
$$

It can be verified that $\Lambda_{1}$ and $\Lambda_{2}$ lie on the grid generated by the lines $x_{2}=x_{1} \tan \theta_{j}+$ $\frac{\pi}{k_{p} \cos \theta_{j}} n$ for $n \in \mathbb{Z}, j=1,2$. Thus we have $\Lambda_{1}, \Lambda_{2} \in \mathcal{N}_{2}\left(\theta_{1}, k_{p}\right) \cap \mathcal{N}_{2}\left(\theta_{2}, k_{p}\right) \cap \mathcal{D}_{2}\left(\theta_{3}, k_{p}\right)$ with $\theta_{j}(j=1,2,3)$ and $k_{p}$ chosen above. Set

$$
\begin{aligned}
& u^{(1)}(x)=\left(\begin{array}{c}
4 / 5 \\
-3 / 5
\end{array}\right) e^{i\left(10 x_{1}-\frac{15}{2} x_{2}\right)}+\left(\begin{array}{c}
-4 / 5 \\
3 / 5
\end{array}\right) e^{i\left(-10 x_{1}+\frac{15}{2} x_{2}\right)}, \\
& u^{(2)}(x)=\left(\begin{array}{c}
-3 / 5 \\
-4 / 5
\end{array}\right) e^{i\left(-\frac{15}{2} x_{1}-10 x_{2}\right)}+\left(\begin{array}{c}
3 / 5 \\
4 / 5
\end{array}\right) e^{i\left(\frac{15}{2} x_{1}+10 x_{2}\right)}, \\
& u^{(3)}(x)=\left(\begin{array}{c}
7 / 25 \\
-24 / 25
\end{array}\right) e^{i\left(\frac{7}{2} x_{1}-12 x_{2}\right)}+\left(\begin{array}{c}
-7 / 25 \\
24 / 25
\end{array}\right) e^{i\left(-\frac{7}{2} x_{1}+12 x_{2}\right)}+\left(\begin{array}{l}
1 \\
0
\end{array}\right) e^{i \frac{25}{2} x_{1}}+\left(\begin{array}{c}
-1 \\
0
\end{array}\right) e^{-i \frac{25}{2} x_{1}} .
\end{aligned}
$$


Then each vector function $u^{(j)}(j=1,2,3)$ satisfies the Navier equation in the whole plane, the quasi-periodicity condition and the boundary conditions of the third kind on both $\Lambda_{1}$ and $\Lambda_{2}$.

Corollary 6. Suppose that the assumptions of Theorem 5 are satisfied. Then we have

(i) If (44) holds for three incident pressure waves with distinct incident angles, then either $\Lambda_{1}=\Lambda_{2}$, or

$$
\Lambda_{1}, \Lambda_{2} \in \mathcal{N}_{2}\left(\theta, k_{p}\right) \cap \mathcal{N}_{2}\left(\theta-\frac{\pi}{2}, k_{p}\right) \cap \mathcal{D}_{2}\left(2 \theta-\frac{\pi}{2}, k_{p}\right),
$$

where $\theta \in\left(0, \frac{\pi}{2}\right)$ is one of the three incident angles. Moreover, $2 k_{p} \sin \theta \in$ $\mathbb{Z}, 2 k_{p} \cos \theta \in \mathbb{Z}, 4 k_{p}\left(\cos ^{2} \theta-1\right) \in \mathbb{Z}$ and $2 k_{p} \in \mathbb{Z}$. In addition, a Rayleigh frequency of the compressional part occurs for the incident angle $2 \theta-\frac{\pi}{2}$.

(ii) If (44) holds for four incident pressure waves with distinct incident angles, then $\Lambda_{1}$ and $\Lambda_{2}$ must be identical, while three incident waves are always enough to imply $\Lambda_{1}=\Lambda_{2}$ if Rayleigh frequencies of the compressional part for each incident angle are excluded.

(iii) Given the a priori information that $\Lambda \in \mathcal{A}$ has one corner point at the origin and is not an element of $\mathcal{N}_{2}\left(\theta, k_{p}\right) \cup \mathcal{D}_{2}\left(\theta, k_{p}\right) \cup \mathcal{N}_{3}\left(\theta, k_{p}\right) \cup \mathcal{N}_{4}\left(0, k_{p}\right)$, the near field data $u\left(x_{1}, b ; \theta\right), x_{1} \in(0,2 \pi)$, from one incident pressure wave with the incident angle $\theta$ are enough to identify $\Lambda$ uniquely.

We refer to [14, Corollary 5] for additional conditions on the incident angles $\theta_{j}$ and the compressional wave number $k_{p}$ guaranteeing that each grating profile $\Lambda \in \mathcal{A}$ can be uniquely determined by three incident pressure waves under the third kind boundary conditions.

7. Inverse scattering of incident shear waves. In this section, we consider uniqueness for the inverse scattering of the incident shear wave $u^{i n}:=\hat{\theta}^{\perp} \exp \left(i k_{s} x\right.$. $\hat{\theta}$ ) defined in (2). Recalling the representation (35) in Section 6, we introduce the following notations:

$$
\begin{aligned}
& (\alpha,-\gamma):=\left(\alpha_{0},-\gamma_{0}\right)=\left(k_{s} \sin \theta,-k_{s} \cos \theta\right)=k_{s} \hat{\theta}^{\top} . \\
& P:=\left\{n \in \mathbb{Z}:\left|\alpha_{n}\right| \leq k_{p}, A_{p, n} \neq 0\right\}, S:=\left\{n \in \mathbb{Z}:\left|\alpha_{n}\right| \leq k_{s}, A_{s, n} \neq 0\right\} \cup\{\kappa\} . \\
& \mathbf{P}_{n}=\left(\alpha_{n}, \beta_{n}\right)^{\top} \text { for } n \in P, \mathbf{S}_{n}=\left(\alpha_{n}, \gamma_{n}\right)^{\top} \text { for } n \in S \backslash\{\kappa\}, \mathbf{S}_{\kappa}=(\alpha,-\gamma)^{\top}=k_{s} \hat{\theta}, \\
& \quad A_{s, \kappa}=\frac{1}{k_{s}} .
\end{aligned}
$$

In this case, $\mathcal{S}$ consists of finitely many upward propagating directions of the shear part of $u,\left\{\mathbf{S}_{n}: n \in S \backslash\{\kappa\}\right\}$, as well as the incident direction $\mathbf{S}_{\kappa}$, while $\mathcal{P}$ consists of the directions of the compressional part, $\left\{\mathbf{P}_{n}: n \in P\right\}$. Since the plane shear wave of the form (2) is taken as an incident wave, all elements in $\mathcal{P}$ and $\mathcal{S}$ but $\mathbf{S}_{\kappa}$ have a non-negative second component. There are at most two elements in $\mathcal{S},\left(k_{s}, 0\right)$ and $\left(-k_{s}, 0\right)$, that have vanishing $x_{2}$-components if $\pi_{s} \neq \emptyset$, while $\left(k_{p}, 0\right)$ or $\left(-k_{p}, 0\right)$ belongs to $\mathcal{P}$ if $\pi_{p} \neq \emptyset$. Based on these facts and the reflection principle, the set $D$, which consists of the third or fourth kind lines that pass through the origin, also forms an equi-angular system of lines in $\mathbb{R}^{2}$, and the rotational invariance of $\mathcal{P}$ and $\mathcal{S}$ can be proved by an argument similar to that used in Section 6 . In addition, the relation $\mathcal{P}=\emptyset$ can be derived from the rotational invariance and the geometric assumption that $\Lambda \in \mathcal{A}$ is given by the graph of a function. Thus, with necessary modifications related to the incident shear wave, all the arguments in Section 6 can be carried over to the case of inverse scattering of incident shear waves. This leads to the following lemma, which can be used to determine the propagating directions of the shear part as well as the structure of the grating profile around a corner point. 
LEMMA 12. Let the incident wave be the incident shear wave defined in (2), and suppose the assumptions (A2) and (A3) at the beginning of Section 6 hold. We have

(i) Under the boundary conditions of the third kind,

$$
D^{\#}=2, \pi_{s} \neq \emptyset, D=\left\{L_{\frac{\theta}{2}+\frac{\pi}{4}}, L_{\frac{\theta}{2}-\frac{\pi}{4}}\right\}, \mathcal{S}=\left\{(\alpha,-\gamma),(-\alpha, \gamma),\left(k_{s}, 0\right),\left(-k_{s}, 0\right)\right\}
$$

(ii) Under the boundary conditions of the fourth kind, $2 \leq D^{\#} \leq 4$. Furthermore, if $D^{\#}=2$ and $\pi_{s}=\emptyset$, then

$$
D=\left\{L_{\theta}, L_{\theta+\frac{\pi}{2}}\right\}, \mathcal{S}=\{(\alpha,-\gamma),(-\alpha, \gamma)\}
$$

if $D^{\#}=2$ and $\pi_{s} \neq \emptyset$, then

$$
D=\left\{L_{\frac{\theta}{2}+\frac{\pi}{4}}, L_{\frac{\theta}{2}-\frac{\pi}{4}}\right\}, \mathcal{S}=\left\{(\alpha,-\gamma),(-\alpha, \gamma),\left(k_{s}, 0\right),\left(-k_{s}, 0\right)\right\}
$$

if $D^{\#}=3$, then

$$
D=\left\{L_{\theta-\frac{\pi}{2}}, L_{\theta+\frac{\pi}{6}}, L_{\theta+\frac{5 \pi}{6}}\right\}, \mathcal{S}=\left\{\boldsymbol{S}_{\kappa}, \operatorname{Rot}_{\frac{2 \pi}{3}}\left(\boldsymbol{S}_{\kappa}\right), \operatorname{Rot}_{\frac{4 \pi}{3}}\left(\boldsymbol{S}_{\kappa}\right)\right\} ;
$$

if $D^{\#}=4$, then

$$
\theta=0, D=\left\{L_{0}, L_{\frac{\pi}{4}}, L_{-\frac{\pi}{4}}, L_{\frac{\pi}{2}}\right\}, \mathcal{S}=\left\{\left(0, k_{s}\right),\left(-k_{s}, 0\right),\left(k_{s}, 0\right),\left(0,-k_{s}\right)\right\}
$$

We finally summarize the uniqueness results in the following theorems.

TheOREM 7. Let $\Lambda_{1}, \Lambda_{2} \in \mathcal{A}$ such that one corner point of $\Lambda_{1}$ or $\Lambda_{2}$ is located at the origin. Assume the boundary conditions of the third kind are imposed on $\Lambda_{j}, j=$ 1,2. If the relation (44) holds for one incident shear wave with incident angle $\theta \in$ $\left(-\frac{\pi}{2}, \frac{\pi}{2}\right)$, then one of the following cases must occur:

(i) $\Lambda_{1}=\Lambda_{2}$.

(ii) $\Lambda_{1}, \Lambda_{2} \in \mathcal{D}_{2}\left(\theta, k_{s}\right), \pi_{s}=\emptyset$, and the total field takes the form

$$
\begin{aligned}
u(x)= & \hat{\theta}^{\perp} \exp \left(i k_{s} x \cdot \hat{\theta}\right)-\hat{\theta}^{\perp} \exp \left(-i k_{s} x \cdot \hat{\theta}\right) \\
& +e_{2} \exp \left(i k_{s} x_{1}\right)-e_{2} \exp \left(-i k_{s} x_{1}\right) \text { in } \mathbb{R}^{2},
\end{aligned}
$$

where $u=u_{j}(j=1,2), e_{2}=(0,1)^{\top}$.

TheOREM 8. Let $\Lambda_{1}, \Lambda_{2} \in \mathcal{A}$ be such that one of the profiles $\Lambda_{1}, \Lambda_{2}$ has a corner point at the origin. Assume the fourth kind boundary conditions are imposed on $\Lambda_{j}, j=1,2$. If the relation (44) holds for one incident shear wave with incident angle $\theta \in\left(-\frac{\pi}{2}, \frac{\pi}{2}\right)$, then one of the following cases must occur:

(i) $\Lambda_{1}=\Lambda_{2}$.

(ii) (a) $\Lambda_{1}, \Lambda_{2} \in \mathcal{N}_{2}\left(\theta, k_{s}\right), \pi_{s}=\emptyset$, and the total field takes the form

$$
u(x)=\hat{\theta}^{\perp} \exp \left(i k_{s} x \cdot \hat{\theta}\right)-\hat{\theta}^{\perp} \exp \left(-i k_{s} x \cdot \hat{\theta}\right) \quad \text { in } \quad \mathbb{R}^{2} .
$$

(b) $\Lambda_{1}, \Lambda_{2} \in \mathcal{D}_{2}\left(\theta, k_{s}\right), \pi_{s} \neq \emptyset$, and the total field takes the form

$$
\begin{aligned}
u(x)= & \hat{\theta}^{\perp} \exp \left(i k_{s} x \cdot \hat{\theta}\right)-\hat{\theta}^{\perp} \exp \left(-i k_{s} x \cdot \hat{\theta}\right) \\
& -e_{2} \exp \left(i k_{s} x_{1}\right)+e_{2} \exp \left(-i k_{s} x_{1}\right) \text { in } \mathbb{R}^{2} .
\end{aligned}
$$


(iii) $\Lambda_{1}, \Lambda_{2} \in \mathcal{N}_{3}\left(\theta, k_{s}\right)$ with $\theta \in\left[-\frac{\pi}{6}, \frac{\pi}{6}\right]$. In this case, $\pi_{s} \neq \emptyset$ if $\theta=\frac{\pi}{6}$ or $\theta=-\frac{\pi}{6}$, and the total field takes the following form in $\mathbb{R}^{2}$ :

$$
\begin{aligned}
u(x)= & \hat{\theta}^{\perp} \exp \left(i k_{s} x \cdot \hat{\theta}\right)+\left(\operatorname{Rot}_{\frac{2 \pi}{3}}(\hat{\theta})\right)^{\perp} \exp \left(i k_{s} x \cdot \operatorname{Rot}_{\frac{2 \pi}{3}}(\hat{\theta})\right) \\
& +\left(\operatorname{Rot}_{\frac{4 \pi}{3}}(\hat{\theta})\right)^{\perp} \exp \left(i k_{s} x \cdot \operatorname{Rot}_{\frac{4 \pi}{3}}(\hat{\theta})\right) .
\end{aligned}
$$

(iv) $\Lambda_{1}, \Lambda_{2} \in \mathcal{N}_{4}\left(0, k_{s}\right), \theta=0, \pi_{s} \neq \emptyset$, and the total field takes the form

$$
\begin{aligned}
u(x)= & e_{1} \exp \left(-i k_{s} x_{2}\right)-e_{1} \exp \left(i k_{s} x_{2}\right) \\
& +e_{2} \exp \left(i k_{s} x_{1}\right)-e_{2} \exp \left(-i k_{p} x_{1}\right) \text { in } \mathbb{R}^{2} .
\end{aligned}
$$

Here $u=u_{j}(j=1,2), e_{1}=(1,0)^{\top}$ and $e_{2}=(0,1)^{\top}$.

Note that the two basic results presented in the introduction follow from Remark 7 (ii) and (iii), Corollary 6 (ii) and the above Theorems 7 and 8. The counterexamples in the case of the incident shear wave can be constructed similarly as those in Sections 6.2 and 6.3 .

Acknowledgment. The second author gratefully acknowledges the support by the German Research Foundation (DFG) under Grant No. EL 584/1-1. The authors would also like to thank Prof. Zou Jun at the Chinese University of Hong Kong and Zhang Hai at the Michigan State University for personal communications and sending us the manuscripts $[9,10]$.

\section{REFERENCES}

[1] H. AmmaRI, Uniqueness theorems for an inverse problem in a doubly periodic structure, Inverse Problems, 11 (1995), pp. 823-833.

[2] C. Antonios, G. Drossos, and K. Kiriakie, On the uniqueness of the inverse elastic scattering problem for periodic structures, Inverse Problems, 17 (2001), pp. 1923-1935.

[3] T. AREns, The scattering of plane elastic waves by a one-dimensional periodic surface, Math. Meth. Appl. Sci., 22 (1999), pp. 55-72.

[4] T. AREns, A new integral equation formulation for the scattering of plane elastic waves by diffraction gratings, J. Integral Equations Appl., 11 (1999), pp. 275-297.

[5] T. Arens, Uniqueness for elastic wave scattering by rough surfaces, SIAM J. Math. Anal., 33 (2001), pp. 461-471.

[6] T. Arens, Existence of solution in elastic wave scattering by unbounded rough surfaces, Math. Meth. Appl. Sci., 25 (2002), pp. 507-528.

[7] G. BAO, A uniqueness theorem for an inverse problem in periodic diffractive optics, Inverse Problems, 10 (1994), pp. 335-340.

[8] G. Bao, L. Cowsar, and W. eds. Masters, Mathematical Modeling in Optical Science, 2001 (Philadelphia: SIAM).

[9] G. BAO, H. ZhANG, AND J. Zou, Unique determination of periodic polyhedral structures by scattered electromagnetic fields, Trans. Amer. Math. Soc., 363 (2010), pp. 4527-4551.

[10] G. BAO, H. Zhang, AND J. Zou, Unique determination of periodic polyhedral structures by scattered electromagnetic fields $\mathbb{I}$, in preparation, 2010.

[11] G. BAO AND Z. Zhou, An inverse problem for scattering by a doubly periodic structure, Trans. Amer. Math. Soc., 350 (1998), pp. 4089-4103.

[12] A. Böttcher and B. Silbermann, Analysis of Toeplitz Operators, 1989 (Berlin: Akademie).

[13] J. Elschner AND G. Hu, Variational approach to scattering of plane elastic waves by diffraction gratings, Math. Meth. Appl. Sci., 33 (2010), pp. 1924-1941.

[14] J. Elschner AND G. Hu, Global uniqueness in determining polygonal periodic structures with a minimal number of incident plane waves, Inverse Problems, 26 (2010), 115002.

[15] J. Elschner, G. Schmidt, And M. Yamamoto, An inverse problem in periodic diffractive optics: global uniqueness with a single wavenumber, Inverse problems, 19 (2003), pp. 779 787.

[16] J. Elschner and M. Yamamoto, Uniqueness in determining polygonal periodic structures, Z. Anal. Anwend., 26 (2007), pp. 165-177. 
[17] J. Elschner And M. YAmamoto, Uniqueness in inverse elastic scattering with finitely many incident waves, Inverse Problems, 26 (2010).

[18] F. Hettlich AND A. KIRSCH, Schiffer's theorem in inverse scattering for periodic structures, Inverse Problems, 13 (1997), pp. 351-361.

[19] A. KIRSCH, Uniqueness theorems in inverse scattering theory for periodic structures, Inverse Problems, 10 (1994), pp. 145-152.

[20] V. D. Kupradze Et AL., Three-dimensional Problems of the Mathematical Theory of Elasticity and Thermoelasticity, 1979 (Amsterdam: North-Holland).

[21] H. Liu, M. Yамамото, And J. Zou, Reflection principle for Maxwell equations and its application to inverse electromagnetic scattering, Inverse Problems, 23 (2007), pp. 2357-2366.

[22] H. Liu, M. Yамамото, AND J. Zou, New reflection principles for Maxwell equations and their applications, Numer. Math. Theory Methods Appl., 2 (2009), pp. 1-17.

[23] H. LIU AND J. ZOU, Uniqueness in an inverse acoustic obstacle scattering problem for both sound-hard and sound-soft polyhedral scatterers, Inverse Problems, 23 (2006), pp. 515-524. 
